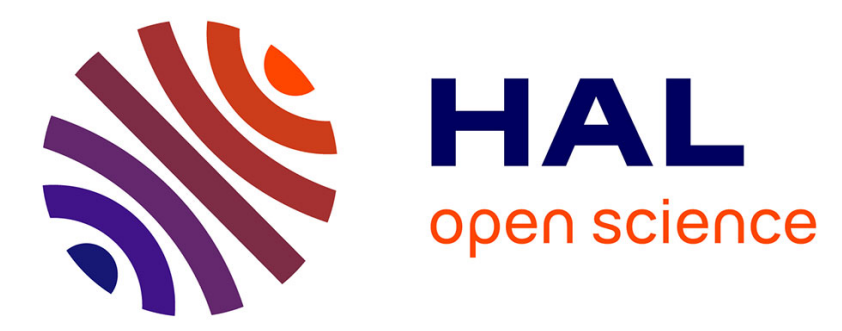

\title{
Participation budgétaire et performance managériale : lien non significatif et contingences
}

\author{
Adrien B. Bonache, Jonathan Maurice, Karen Moris
}

\section{To cite this version:}

Adrien B. Bonache, Jonathan Maurice, Karen Moris. Participation budgétaire et performance managériale: lien non significatif et contingences. Comptabilité - Contrôle - Audit, 2012, 18 (2), pp.125184. 10.3917/cca.182.0125. hal-00736241

\section{HAL Id: hal-00736241 \\ https://hal.science/hal-00736241}

Submitted on 12 Nov 2019

HAL is a multi-disciplinary open access archive for the deposit and dissemination of scientific research documents, whether they are published or not. The documents may come from teaching and research institutions in France or abroad, or from public or private research centers.
L'archive ouverte pluridisciplinaire HAL, est destinée au dépôt et à la diffusion de documents scientifiques de niveau recherche, publiés ou non, émanant des établissements d'enseignement et de recherche français ou étrangers, des laboratoires publics ou privés. 


\title{
Participation budgétaire et performance managériale : Lien non significatif et contingences Budgetary participation and managerial performance: Non significant link and contingencies
}

\author{
Adrien B. BONACHE*, Jonathan MAURICE** et Karen MORIS***
}

* ATER, Université de Bourgogne, LEG-Fargo - UMR 5118 (CNRS)

** ATER, Université Montpellier 1, MRM - EA 4557

*** Enseignant-chercheur, Université de Strasbourg - EM Strasbourg, LaRGE - EA 2364

Pour citer cet article :

BONACHE (Adrien), MAURICE (Jonathan) et MORIS (Karen), «Participation budgétaire et performance managériale : lien non significatif et contingences », Comptabilité - Contrôle - Audit, septembre 2012, t. 18, vol. 2, p. 125-184 ; <http://dx.doi.org/10.3917/cca.182.0125>.

\section{Résumé}

Cet article met en œuvre trois métaanalyses pour étudier le lien entre la participation budgétaire et la performance managériale. Il permet de mettre en application certaines préconisations du forum international de Potsdam de 1994 sur la métaanalyse, dans le domaine du contrôle de gestion. Après une présentation des techniques de méta-analyse utilisées et des articles compilés, trois méta-analyses ont été réalisées sur le lien entre la participation budgétaire et la performance managériale. Nous mettons notamment en œuvre la «synthèse des meilleures études », un type de méta-analyse préconisé par Slavin (1995). Cette métaanalyse, davantage pertinente, amène à conclure globalement à l'existence d'un lien non significatif entre la participation budgétaire et la performance managériale. Nous discutons alors de la présence de contingences culturelles et temporelles pour expliquer l'hétérogénéité des résultats obtenus dans les différentes études.

Mots-ClÉS : PARTICIPATION BUdGÉTAIRE PERFormance MANAGÉRIALE - MÉTAANALYSE - SYNTHÈSE DES MEILLEURES ÉTUDES - MÉTHODES DES VOTES.

\section{Abstract}

Through a combination of three metaanalyses this paper aims at studying the link between budgetary participation and managerial performance. It will make use of some recommendations suggested during the 1994 Potsdam International Congress on meta-analysis, in the field of management control. After an overview of the methods used for meta-analyses and a review of the literature, three meta-analyses studying the link between budgetary participation and managerial performance were carried out. Following Slavin's (1995) suggestions, we have implemented the "best evidence synthesis" method that appears to be the most relevant. It allows us to conclude, on the whole, that budgetary participation has no significant impact on managerial performance. Finally cultural and temporal contingencies have also been discussed so as to explain the heterogeneity resulting from these different studies.

KEYWORDS: BUDGETARY PARTICIPATION MANAGERIAL PERFORMANCE - META-ANALYSIS BEST EVIDENCE SYNTHESIS - BOX SCORE METHODS. 
Correspondance :

Adrien B. BONACHE

2, boulevard Gabriel

BP 26611

21066 Dijon CEDEX

bonache@ens-cachan.fr

Karen MORIS

EM Strasbourg

61, avenue de la Forêt

Noire

67085 Strasbourg

CEDEX

karenmoris@yahoo.fr
Jonathan MAURICE

Espace Richter - Bât. B

Rue Vendémiaire

CS 19519

34960 Montpellier CEDEX 2

jonathan.maurice@ens-cachan.org

Remerciements :

Les auteurs tiennent à remercier les trois réviseurs anonymes pour leurs remarques et conseils; Benjamin Dreveton, Didier Le Maître et les participants du $30^{\mathrm{e}}$ congrès de l'AFC pour leurs suggestions d'amélioration de la première version de l'article ; Kar-Ming Chong, Alan S. Dunk, Irène Georgescu, Lucie Magnien, Feng-Yu Ni et Jeffrey J. Quirin pour leur aide dans la complétion de la base de données d'articles; Axelle Akermann pour ses suggestions d'amélioration de la dernière version de l'article.

\section{Introduction}

Un manager participant à la détermination de ses ressources budgétaires est-il un manager plus performant? Si la question n'est pas nouvelle, elle reste d'importance dans le domaine académique lorsqu'on observe l'évolution de la littérature. De 1963 à 2010, plus d'une centaine d'études se sont penchées sur la question, dont plus d'un tiers dans les cinq dernières années. Malgré ces nombreuses recherches, la réponse à la question demeure controversée (Nouri et Kyj 2008), à la fois sur le plan théorique et sur le plan empirique.

Face à cette absence de consensus, de nombreux chercheurs ont étudié l'impact d'un grand nombre de facteurs de contingences sur le lien entre la participation budgétaire et la performance managériale : satisfaction au travail (Frucot et Shearon 1991), style de leadership (Brownell 1983), culture (Lau et al. 1997 ; Su 2001 ; Leach-López et al. 2007 ; Leach-López et al. 2008 ; Leach-López et al. 2009), motivation (Brownell et McInnes 1986), difficulté de la tâche (Mia 1989 ; Brownell et Dunk 1991; Lau et al. 1995, 1997), volatilité et information pertinente sur le travail (Kren 1992), mode de participation (Lindquist 1995 ; Libby 1999; Byrne et Damon 2008), ambiguïté de rôle (Chenhall et Brownell 1988 ; Nouri et Parker 1998), taille des firmes (Ni et Su 2001), difficulté des objectifs budgétaires (Ni et al. 2005), mise en valeur du budget dans l'organisation (Lau et Lim 2002b), expérience des managers (Heath et Brown 2007) et place de l'innovation dans l'organisation (Dunk 1995a). D'autres chercheurs ont renversé la causalité du lien pour montrer que la performance pouvait aussi déterminer le degré de participation (Nouri et Kyj 2008). 
En dépit de ces études, la question du lien entre la participation budgétaire et la performance managériale demeure ouverte et une synthèse de cette littérature foisonnante semble nécessaire. Une méthode de synthèse intéressante est la méta-analyse. En cas de résultats apparemment discordants, une méta-analyse permet d'obtenir une vue globale et quantifiée de l'effet d'une variable sur une autre (Cucherat et al. 1997). Dans la littérature, il existe trois études utilisant un certain type de métaanalyse sur le lien participation budgétaire - performance managériale. Mais aucune ne porte sur l'ensemble de la littérature disponible. La première méta-analyse (Greenberg et al. 1994) est ancienne et comprend seulement un tiers de la littérature actuelle : onze études utilisant comme méthode d'investigation un sondage et vingt-neuf études utilisant une expérimentation. La deuxième métaanalyse (Derfuss 2009) ne concerne pas seulement l'étude du lien entre la participation et la performance et n'intègre que vingt-huit études sur plus de cent disponibles actuellement sur le sujet : les études publiées en langue anglaise avant 2007 dans des revues classées (en Allemagne), dans les ouvrages de la série Advances in ou disponibles sur les bases Science Direct ou EBSCO / Business Source Premier et n'utilisant que certaines techniques statistiques. La troisième méta-analyse (Bonache et al. 2010) est spécifique à trois pays sur lesquels plusieurs études, sélectionnant aléatoirement leur échantillon, sont disponibles : seules quinze études sont prises en compte. Par conséquent, la totalité de la littérature actuelle concernant le lien entre la participation budgétaire et la performance managériale n'a pas encore été synthétisée de façon adéquate et complète. L'objet de cet article est de combler ce manque en combinant différentes méthodes de méta-analyse sur la littérature du domaine la plus complète et actualisée possible. Cette combinaison de plusieurs techniques de méta-analyse permet alors d'allier les avantages et de pallier les principaux inconvénients de chacune d'entre elles.

Suivant Merchant et Otley (2007), cette démarche se base sur l'hypothèse que la recherche en contrôle de gestion permet de mettre en avant des "résultats universels » et des contingences éventuelles autour de ces «résultats universels », et non uniquement des contingences sans résultat universel. Dans cet article, nous mettons en œuvre trois méta-analyses :

1. Une méta-analyse non quantitative permettant d'inclure et de traiter l'ensemble de la littérature au détriment du contrôle de la qualité des études incluses et de la quantification de l'ampleur de l'effet moyen d'une variable sur une autre ;

2. Une méta-analyse quantitative, permettant de calculer l'ampleur de cet effet et une prise en compte de la qualité des études incluses, à partir des études pouvant faire l'objet d'un tel traitement ;

3. Une best evidence synthesis : une méta-analyse quantitative ne prenant en compte que les études de très bonne qualité au regard de trois critères : utilisation d'échelles de mesure ayant un alpha de Cronbach élevé, tirage aléatoire de l'échantillon et publication dans des revues classées.

L'usage de différentes méthodes de méta-analyse permet de s'assurer que les résultats positifs et significatifs mis en avant dans des études précédentes (Greenberg et al. 1994 ; Derfuss 2009), ne sont pas uniquement dus à l'usage d'une méta-analyse quantitative particulière (celle de Hunter et Schmidt [2004]) pour synthétiser des résultats publiés dans les seules revues anglo-saxonnes. La sensibilité des résultats aux études sélectionnées et à la méthode retenue reste un point important. Bonache et al. (2010) ont trouvé, avec un autre type de méta-analyse quantitative, un résultat non significatif et négatif en ne retenant que des études sélectionnant aléatoirement leur échantillon et n'utilisant que les échelles de mesure de la participation et de la performance ayant l'alpha de Cronbach le plus élevé. 
Dans cet article, la comparaison des techniques de méta-analyses, appliquées à l'ensemble de la littérature disponible, permet de répondre à la question de recherche suivante : l'impact de la participation budgétaire sur la performance managériale est-il significativement positif?

L'article est organisé de la façon suivante. La section 1 introduit la littérature sur le lien entre la participation budgétaire et la performance managériale ainsi que les articles synthétisés. La section 2 présente les principes, méthodes et résultats obtenus des méta-analyses non quantitatives : elles permettent de synthétiser tous les articles, mais ne donnent pas une estimation de l'effet entre les deux variables étudiées. La section 3 introduit alors les principes, méthodes et résultats obtenus d'une métaanalyse quantitative exhaustive : cette technique donne une estimation de l'effet mais inclut des articles de faible qualité. En conséquence, la section 4 détaille les critères de sélection utilisés pour faire une méta-analyse quantitative sur les articles « de qualité » et les résultats obtenus avec cette méthode de synthèse sélective. La section 5 compare les résultats de cette méthode de synthèse aux trois précédentes méta-analyses de la littérature (Greenberg et al. 1994 ; Derfuss 2009 ; Bonache et al. 2010). Constatant la présence d'hétérogénéité entre les études de qualité et une sensibilité des résultats aux critères d'inclusion, la section 6 discute les pistes de recherche futures pour expliquer cette hétérogénéité et cette sensibilité : la présence de contingences culturelles et temporelles.

\section{Problématique de la recherche}

Une revue de littérature classique permet de comprendre le débat autour de l'impact de la participation budgétaire sur la performance des managers. À la fois sur le plan théorique et sur le plan empirique, la relation entre la participation budgétaire et la performance managériale apparaît controversée. Cela justifie l'utilisation de la méta-analyse pour tenter de clarifier le débat.

\subsection{Une relation équivoque sur le plan théorique}

Premièrement, nous rappelons les raisons expliquant pourquoi faire participer un manager à l'élaboration du budget de son organisation peut améliorer sa performance. Secondement, nous mettons en évidence les arguments théoriques modérant cet effet positif de la participation sur la performance. Le débat reste ainsi ouvert.

\subsubsection{LES EFFETS POSITIFS DE LA PARTICIPATION AU BUDGET}

La littérature en comptabilité et en management souligne souvent l'importance de la participation des managers dans les problématiques d'établissement des budgets. Dans cette littérature, la participation à l'élaboration du budget de managers, se trouvant au milieu ou en bas de la ligne hiérarchique, peut avoir deux effets positifs (Banović 2005). Premièrement, grâce à la participation au budget, les managers en bas de la hiérarchie se sentent plus impliqués. Leur volonté de respecter les objectifs fixés est alors plus grande (Welsch et al. 1988). Deuxièmement, la participation permet de diminuer les asymétries d'information. Les connaissances du terrain étant détenues par ceux qui se trouvent en bas de la hiérarchie (Kang et al. 1994), la participation à l'élaboration du budget permet d'améliorer l'information à destination de leurs supérieurs. 
Enfin, d'après Shields et Shields (1998), la participation des managers est mise en œuvre «pour planifier et établir des buts dans des environnements incertains, pour motiver les subordonnés lorsqu'il y a des incertitudes concernant les tâches à accomplir mais aussi pour coordonner les tâches en cas d'interdépendance entre celles-ci » (traduction des auteurs [désormais T.D.A.] ${ }^{1}$ ). La participation paraît être un remède à certains effets pervers associés à l'élaboration du budget (Argyris 1952, p. 28). Néanmoins, certains chercheurs ont également mis en évidence des facteurs atténuant cet impact positif ou le rendant négatif.

\subsubsection{LE QUESTIONNEMENT AUTOUR DE CES EFFETS POSITIFS}

Le fait que les managers puissent modifier les informations budgétaires à leur avantage ou qu'ils n'aient pas d'influence réelle sur les budgets semble remettre en question les effets positifs de la participation budgétaire sur la performance managériale.

Une limite à la participation budgétaire est la possibilité pour les managers de créer du slack, défini par Walker et Johnson (1999, p. 3) comme « le montant par lequel un manager surestime ses besoins en ressources pour mener à bien ses actions ou sous-estime ses capacités productives dès lors qu'il peut participer à l'élaboration du standard sur lequel est évaluée sa performance » (T.D.A. ${ }^{2}$ ). Le biais informationnel ainsi répercuté dans les budgets peut alors être source d'inefficience lors de l'allocation des ressources de l'organisation (Magee 1980; Christensen 1982). Même si cette propension au slack peut être modérée par la pression sociale (Young 1985, p. 830), elle n'en demeure pas moins une source potentielle de gaspillage pouvant réduire voire annuler les bénéfices attendus de la participation.

De plus, la participation peut ne pas être « réelle ». Dans le cadre de l'élaboration des budgets, les subordonnés n'ont pas toujours la possibilité d'influencer concrètement l'élaboration des budgets (Becker et Green 1962, 1964), même s'ils participent au processus d'élaboration. Cela peut diminuer alors les effets positifs de la participation.

Ensuite, si la participation permet de modifier les budgets à l'avantage des subordonnés ou de créer un effet «Hawthorne» (Roethlisberger et Dickson 1939) en ne donnant qu'une illusion de participation, ses effets positifs à long terme peuvent être affectés. Cela peut même avoir un impact négatif sur le résultat de l'entreprise (Penno 1990).

En résumé, selon ces différents travaux, la participation budgétaire n'aurait a priori pas un impact clair sur la performance managériale. Un recensement des résultats empiriques ne semble pas, non plus, donner une réponse univoque.

\subsection{Une relation équivoque sur le plan empirique}

Le consensus semble fragile en ce qui concerne le lien entre participation et performance, et la question du signe de ce lien reste aujourd'hui encore ambiguë (Nouri et Kyj 2008). Après les premières études comme celle de Milani (1975) montrant un lien positif, d'autres transforment la représentation de cette relation : le lien serait modifié par des variables contextuelles.

Par exemple, lors d'une expérience avec 46 étudiants en comptabilité, Brownell (1981) trouve que 
ce lien dépend de la personnalité du manager : la participation accroîtrait la performance managériale, seulement pour ceux pensant avoir leur destin en main. Dans ce même article, il corrobore ce résultat par une expérience identique réalisée sur 48 managers de la région de San Francisco et en accroît alors la validité externe ${ }^{3}$. De nombreux chercheurs ont également testé d'autres facteurs de contingence, comme la motivation (Brownell et McInnes 1986), l'ambiguïté de rôle (Chenhall et Brownell 1988 ; Nouri et Parker 1998) ou la culture (Lau et al. 1997 ; Su 2001 ; Leach-López et al. 2007 ; Leach-López et al. 2008 ; Leach-López et al. 2009).

Suivant Brownell (1981, 1982a, 1982b), certains auteurs cherchent une explication contingente de ce lien entre participation budgétaire et performance managériale pour expliquer le faible degré de signification des résultats. Nouri et Parker (1998) montrent notamment que l'implication organisationnelle est un facteur de contingence. Frucot et Shearon (1991) et Tsui (2001) soulignent l'importance des facteurs culturels dans cette relation. Enfin, la perception de justice et l'implication dans les buts modifient l'impact de la participation sur la performance (Wentzel 2002).

Nonobstant, d'autres auteurs, ne trouvant pas de relations significatives, pensent que ce lien est plus complexe (Shields et Young 1993). Par exemple, Kren (1992) et Chong et Chong (2002a) montrent que la participation ne joue sur la performance qu'en ayant un impact sur l'implication, qui elle-même influence l'information pertinente sur le travail (job-relevant information) et cette information affecte, enfin, positivement et significativement la performance.

Finalement, il apparaît qu'une revue de la littérature des travaux empiriques portant sur le lien entre participation budgétaire et performance managériale ne permet pas de conclure de manière univoque sur ce lien, y compris au vu des études récentes. Il semble alors utile de passer par d'autres méthodes de synthèse de la littérature, comme les méta-analyses.

En reprenant la définition de la National Library of Medicine, la méta-analyse est définie par Olkin (1995, p. 133) comme « une méthode quantitative combinant les résultats d'études indépendantes (en principe, tirées de la littérature publiée) et synthétisant les résumés et conclusions qui pourraient servir à évaluer l'efficacité thérapeutique [ou d'une pratique], à planifier des nouvelles études, etc., avec des applications principalement dans le champ de la recherche et la médecine » (T.D.A. ${ }^{4}$ ). Pour réaliser une méta-analyse, il convient de collecter tous les résultats obtenus préalablement sur le sujet. Nous décrivons dans la section suivante la procédure de collecte utilisée.

\subsection{Collecte et présentation des articles compilés}

Nous avons d'abord sélectionné les articles à partir de revues de littérature (Shields et Shields 1998 ; Chalos et Poon 2001 ; Banović 2005) et de méta-analyses (Greenberg et al. 1994 ; Derfuss 2009 ; Bonache et al. 2010) déjà réalisées sur le lien entre participation budgétaire et performance managériale. Dans un premier temps, ces sources ont été complétées par l'intermédiaire des bases ABI Inform, Business Source Premier, JSTOR et Scholar Google avec les expressions «budgetary participation » et «managerial performance » en termes de mots-clefs de recherche. Dans un second temps, ces deux expressions ont été traduites en allemand, chinois, espagnol, français et indonésien pour collecter d'autres articles dans ces mêmes bases.

Puis, notre document de travail a été publié sur Ideas RePEc; il a ensuite été référencé sur Scholar 
Google. Cette procédure, répétée à chaque modification de l'article, a permis de connaître les articles manquants grâce à l'algorithme de référencement de Scholar Google.

Finalement, l'échantillon se compose de 108 articles (cf. annexe 1), écrits en anglais, chinois, français, indonésien ou japonais (cf. section 5 : tableau 5 et tableaux des annexes), excluant certaines études ne fournissant pas de statistiques utilisables (Chong et Johnson 2007 ; Adler et Reid 2008 ; Alim 2008 ; Anttila et Vasic 2009), une étude dont les statistiques sont peu fiables (Hehanussa 2001), trois études ne traitant pas de l'effet direct de la participation budgétaire sur la performance managériale (Murray 1990; Dunk 1995b; Fahrianta 2009) et deux expérimentations traitant de l'impact de la difficulté des objectifs fixés sur la performance (Locke et Bryan 1967 ; Ivancevich 1977).

Sur l'ensemble des études restantes (108), les mesures de la performance et de la participation sont parfois divergentes (cf. annexe 1). Les méthodes et les traitements statistiques utilisés ne sont également pas homogènes. Ces différences compliquent le travail de synthèse des résultats. Ainsi, pour pouvoir compiler tous ces travaux, une méta-analyse non quantitative a d'abord été réalisée.

\section{Mise en œuvre d'une méta-analyse non quantitative}

La méta-analyse est une forme de synthèse visant à pallier les limites des revues de littérature classiques en cherchant à respecter trois principes (Cucherat et al. 1997) : exhaustivité, quantification de l'effet et sélection argumentée des études. Cependant, ces trois principes semblent mutuellement exclusifs. Notamment si l'on sélectionne les études, on ne peut prétendre à l'exhaustivité. Dans cette section 2, nous présentons le premier principe d'exhaustivité avant d'entrer dans le détail technique des méthodes de méta-analyse non quantitatives permettant de respecter ce principe.

\subsection{Premier principe de la méta-analyse : exhaustivité des études analysées}

Selon Slavin (1995), les revues de littérature sont rarement exhaustives et parfois biaisées par la tendance des auteurs à mettre en avant seulement certains articles ${ }^{5}$. De plus, l'auteur ajoute que la procédure de recherche d'articles est, dans ces revues de littérature, rarement décrite et souvent non systématique. Le premier principe de la méta-analyse va alors permettre de pallier ces limites en cherchant l'exhaustivité des études synthétisées, par la systématisation du processus de recherche et la non-sélection a priori des supports de publication des études. Cette procédure est mise en œuvre pour tenter d'échapper au biais de publication ${ }^{6}$ pouvant masquer une réalité de terrain.

Les méta-analyses non quantitatives sont « des techniques de synthèse qui ne s'intéressent pas à la taille de l'effet [d'une variable sur une autre], mais seulement à son existence » (Cucherat et al. 1997, p. 267). Ce type de méta-analyse comprend notamment la méthode des votes et les techniques de combinaison des degrés de signification. La première étudie la prépondérance des études montrant un lien positif et significatif entre deux variables. Les secondes montrent l'existence d'au moins une étude significative. L'avantage de ces méthodes non quantitatives est qu'elles permettent de synthétiser tous les résultats quantitatifs de la littérature, quels que soient les traitements statistiques mis en œuvre et les échelles de mesure utilisées. Elles respectent donc le principe d'exhaustivité. 
En pratique, en dépit de ce principe, les chercheurs n'atteignent pas toujours l'exhaustivité dans la réalisation de méta-analyses. Ils recensent majoritairement les travaux des langues courantes (anglais, français, espagnol, voire allemand), alors qu'il existe aussi des travaux dans d'autres langues. Par exemple, deux précédentes méta-analyses sur le lien participation budgétaire - performance managériale ne retenaient que les articles publiés dans des revues anglo-saxonnes (Greenberg et al. 1994 ; Derfuss 2009). Or, un certain nombre d'études récentes sur le sujet sont disponibles dans des langues «moins lues» dans la mesure où les problématiques budgétaires se diffusent dans des nouveaux pays développés ou en voie de développement, notamment en Asie du Sud-Est. Dans une méta-analyse, ignorer les études faites dans ces pays limite l'intérêt de ce type de méthode visant à synthétiser toute la littérature sur un sujet.

\subsection{Méthodes des méta-analyses non quantitatives}

Compte tenu de la diversité des échelles de mesure et des traitements statistiques utilisés dans les différents articles synthétisés en annexe 1, il a semblé préférable en première analyse de mettre en œuvre des méthodes de méta-analyses non quantitatives. Ce type de méta-analyses permet d'inclure toutes les études empiriques pertinentes disponibles sur le sujet, quelle que soit la méthode d'investigation utilisée, et de voir l'existence d'un lien significatif entre participation budgétaire et performance managériale. D'une part, pour avoir une synthèse exhaustive, la méthode des votes a été utilisée. D'autre part, une technique pertinente de combinaison des degrés de signification a été mobilisée. Par prudence et pour augmenter la pertinence des études incluses, nous avons fait varier le critère d'inclusion et de non-inclusion des études en fonction du degré de signification de leurs résultats. Dans les développements suivants, nous présentons ces deux méthodes plus en détail.

\subsubsection{LA MÉTHODE DES VOTES : CODIFICATION ET TEST DE SIGNE}

La méthode des votes permet de tester s'il y a un lien significatif entre deux variables et d'en connaître son signe. C'est un test binomial de signe. Mais, avant tout traitement statistique, il faut faire un choix en termes de codification des résultats de chaque étude.

\section{CODIFICATION DES RÉSULTATS}

Il existe deux types de codification des résultats des études associés à la méthode des votes.

1. On peut coder les résultats des articles sous forme ternaire : liaison significative positive (1), liaison significative négative $(-1)$ et liaison non significative (n. s.). L'avantage de cette démarche est de permettre l'inclusion dans la méta-analyse de seuils pour la sélection des articles en fonction du degré de signification. Cela permet d'éviter un biais introduit par des articles dont les relations ne sont pas bien établies. Mais elle a l'inconvénient de rejeter les études bien conçues qui n'atteignent pas une puissance suffisante pour que le lien soit significatif, du fait de la faible taille de leur échantillon (Cucherat et al. 1997, p. 5). De plus, l'exclusion de certaines études ne permet pas de montrer le faible degré de signification du résultat de la méta-analyse, puisqu'on s'assure ex ante du degré de signification des études entrant dans l'analyse.

2. Il est aussi possible de coder les résultats en fonction du signe de la relation sans considération pour le degré de signification. Ce second type de codification présente l'avantage de ne pas exclure certaines études présentes dans la littérature.

Une solution de compromis peut être proposée. Il s'agit de privilégier une codification permettant, 
dans notre synthèse de résultats, à la fois de faire entrer tous les résultats concernant le lien participation - performance (on applique alors le second type de codification), et de n'inclure que certains articles (on applique alors le premier type de codification), en faisant varier le degré de sélection $\eta$ ( $p$-value du résultat de l'article $\leq \eta)$. De plus, cette solution permet de tester la stabilité des résultats au regard du degré de signification ( $p$-value). Pour Slavin (1995), ce critère d'inclusion d'une étude donne toutefois trop de poids aux études faites sur de grands échantillons. Selon lui, une méta-analyse doit prendre en compte les études en fonction de leur qualité et non du seul nombre d'individus (qui ne représente qu'une facette, même si importante, de la qualité d'une étude).

\section{LE TEST DES SIGNES}

Le test des signes permet de tester l'hypothèse d'absence de relation significative entre une décision et une variable d'impact. Il s'agit par exemple de diviser le nombre de résultats positifs (et significatifs) par le nombre total de résultats inclus dans la méta-analyse. Si la relation entre les variables est positive, alors la probabilité d'obtenir un résultat significativement positif est supérieure à 0,5 . Le test des signes est donc une application du test non paramétrique binomial aux deux hypothèses suivantes :

$$
\begin{aligned}
& \mathrm{H}_{0}: \pi=0,5, \\
& \mathrm{H}_{1}: \pi>0,5,
\end{aligned}
$$

où $\pi$ est la proportion de résultats positifs estimée, à partir d'un nombre de résultats (significativement) positifs $u$, par $p=u / k$ ( $k$ étant le nombre total d'études). Ce test consiste à calculer, sous $\mathrm{H}_{0}$, la probabilité d'obtenir un nombre de résultats significativement positifs au moins égal à la moitié du nombre de résultats observés. Cette probabilité est égale à la probabilité que la variable aléatoire $\mathrm{U}$ «nombre de résultats positifs » soit supérieure à la valeur observée $u$. Sous $\mathrm{H}_{0}, \mathrm{U}$ suit donc une loi binomiale $\mathrm{B}(k, 1 / 2)$.

\section{ESTIMATION D'UNE VALEUR MOYENNE ET DÉTAIL DU TEST}

L'hypothèse selon laquelle $U$ suit une loi binomiale est valable lorsqu'on combine des résultats dont la taille de l'échantillon est similaire. C'est-à-dire que l'on suppose formellement que $\forall i, n_{i}=n$, où $n_{i}$ est l'effectif ayant permis d'obtenir le résultat de l'article $i$.

Cette hypothèse est particulièrement restrictive parce qu'il est rare qu'un champ de littérature comprenne des résultats basés sur des échantillons de même taille. Nonobstant, Hedges et Olkin (1980) admettent que si les effectifs ne sont pas trop différents, il est possible de les considérer comme étant tous égaux à une « valeur moyenne ». Hedges et Olkin proposent de retenir soit l'effectif moyen, soit l'effectif minimum, soit l'effectif maximum pour estimer cette valeur moyenne. À leur solution, nous préférons celle de Gibbons et al. (1977) du carré de la moyenne des racines des tailles des échantillons (Square Mean Root, désormais SMR) :

$$
\mathrm{SMR}=\bar{n}=\left(\sum_{i=1}^{i=k} \frac{\sqrt{n_{i}}}{k}\right)^{2}
$$

La « valeur moyenne » de Gibbons et al. (1977) présente l'avantage d'être moins influencée par 
les valeurs extrêmes que la moyenne arithmétique. Par exemple, pour les études portant sur la relation entre participation budgétaire et performance managériale, les tailles des échantillons vont de 6 à 358 . Dans ce cas, il est préférable d'utiliser l'estimation de la «valeur moyenne » de Gibbons et al. Il ne nous a donc pas semblé nécessaire d'appliquer les méthodes développées spécialement pour les situations où les échantillons sont très hétérogènes (Cooper et Hedges 1994).

Une fois la question de la valeur moyenne traitée, pour réaliser un test de signe, il convient d'estimer un effet global à partir des résultats sur la base d'un codage dichotomique. Soit $\mathrm{T}_{i}$ l'estimation inconnue de l'effet d'une variable sur une autre du $i$-ème résultat. Si $\mathrm{T}_{i}$ dépasse une variable critique $\mathrm{K}_{\alpha}$ (par exemple $\mathrm{K}_{5} \%$ pour un seuil de signification de $5 \%$ ), ce résultat sera codé significatif-positif et, sinon, non significatif ou négatif. Formellement, nous avons :

$$
\begin{aligned}
& \mathrm{X}_{i}=1 \mathrm{si}_{i}>\mathrm{K}_{\alpha}, \\
& \mathrm{X}_{i}=0 \mathrm{si}_{i} \leq \mathrm{K}_{\alpha} .
\end{aligned}
$$

Nous aboutissons alors au test non paramétrique binomial que nous pouvons exprimer de la façon suivante :

$$
\begin{gathered}
\pi=\mathrm{P}\left(\mathrm{X}_{i}=1\right)=\mathrm{P}\left(\mathrm{T}_{i}>\mathrm{K}_{\alpha}\right), \\
1-\pi=\mathrm{P}\left(\mathrm{X}_{i}=0\right)=\mathrm{P}\left(\mathrm{T}_{i} \leq \mathrm{K}_{\alpha}\right) .
\end{gathered}
$$

Dans une volonté de trianguler les méthodes pour améliorer la validité des conclusions de la métaanalyse non quantitative, outre la méthode des votes, il est possible de mobiliser une méthode permettant de combiner les degrés de signification ( $p$-values).

\subsubsection{COMBINAISON DES DEGRÉS DE SIGNIFICATION}

\section{COMBINAISON DES DEGRÉS DE SIGNIFICATION : INTERPRÉTATION ET PORTÉE LIMITÉE}

La $p$-value, testant la relation d'une variable $\mathrm{X}$ indépendante sur une variable $\mathrm{Y}$ dépendante, est le reflet du test d'une même hypothèse nulle générale : $\mathrm{X}$ n'est pas corrélée avec Y. Soit la relation $\mathrm{Y}_{i}=a_{i} \mathrm{X}_{i}+b_{i}$ pour un article $i$, nous testons ici $\mathrm{H}_{0}: a_{i}=0$.

Ainsi, dans la combinaison des degrés de signification, l'hypothèse nulle (dite « omnibus ») est :

$$
\mathrm{H}_{0}: a_{1}=a_{2}=a_{3}=\ldots=a_{k}=0
$$

Une combinaison des degrés de signification permet de rejeter l'hypothèse omnibus $\mathrm{H}_{0}$ si pour au moins un résultat $i, \mathrm{X}_{i}$ est corrélée avec $\mathrm{Y}_{i}$. L'intérêt d'une combinaison des degrés de signification est alors réduit : elle permet seulement de tester l'existence d'un résultat significatif. Cependant, cette technique a l'avantage de pouvoir regrouper des études envisageant et utilisant des types de critères divers et variés.

Pour cumuler ces degrés de signification, la littérature recense deux types de méthodes (Hedges et Olkin 1985 ; Becker 1994) :

- celles basées sur l'uniformité des distributions des p-values : méthode de la $p$-value minimale et celle de la moyenne des $p$-values; 
- celles basées sur des transformations des p-values : méthode de la somme des logarithmes et celle de la transformation normale.

\section{JUSTIFICATION DU CHOIX DE LA MÉTHODE DE LA MOYENNE DES P-VALUES}

Le problème principal, dans le cas de la combinaison de p-values, est souligné par Becker (1994, p. 227) : «il n'existe pas de test le plus puissant». Cependant, suivant Becker (1994, p. 227), on peut écarter certains tests au profit d'un autre pour notre étude :

- la technique de la somme des logarithmes et celle de la $p$-value minimale sont plus puissantes que la technique de la moyenne des p-values, lorsqu'il n'existe qu'une seule étude atypique. Les résultats présents dans la littérature sur le lien entre participation budgétaire et performance managériale ne semblent pas favoriser l'utilisation de ces tests : il existe plus d'un résultat non significatif et il existe plus d'un résultat significatif. Ces deux tests ne semblent donc pas a priori plus puissants que la technique de la moyenne des $p$-values ;

- il apparaît dans la littérature que le test « de la transformation normale » est plus puissant que celui de la somme des logarithmes, lorsque les tailles d'échantillon et de l'effet sont égales entre les études (Lipták 1958). Concernant la littérature sur le lien entre participation budgétaire et performance managériale, les tailles d'échantillon ne sont pas égales ainsi que les tailles d'effet. Ce test ne semble donc pas plus puissant que celui basé sur la somme des logarithmes et celui basé sur la moyenne des p-values;

- enfin, le test basé sur la moyenne des p-values peut amener à rejeter à tort l'hypothèse nulle, lorsque les variations des $p$-values sont faibles. Dans notre cas, les variations sont assez importantes et nous pouvons donc retenir cette statistique pour faire une combinaison des $p$ values des études sur le lien entre participation budgétaire et performance managériale.

\section{LA MÉTHODE DE LA MOYENNE DES P-VALUES}

Hedges et Olkin (1985) montrent que la statistique suivante suit une loi normale centrée réduite si les degrés de signification $\left(p v_{i}\right.$ de chaque article $i$ ) sont uniformément distribués :

$$
\mathrm{V}=\sqrt{12 \cdot k} \cdot\left(0,5-\sum_{i=1}^{i=k} \frac{p v_{i}}{k}\right) \stackrel{\mathcal{L}}{\rightarrow} \mathcal{N}(0,1)
$$

Cette statistique permet de tester l'hypothèse omnibus à partir d'une somme de $p$-values.

Dans le point suivant, nous décrivons les résultats obtenus avec ces méthodes respectant le principe d'exhaustivité.

\subsection{Résultat d'une méta-analyse exhaustive sur le lien entre participation budgétaire et performance managériale}

Le tableau 1 résume les résultats issus de la méthode des votes, permettant de connaitre le signe du lien entre les deux variables étudiées, et d'une méthode pertinente de combinaison des degrés de signification, afin de savoir s'il existe au moins un résultat significatif. 
Tableau 1

Résultats de la méthode des votes et des combinaisons des p-values

\begin{tabular}{|c|c|c|c|c|}
\hline Études incluses : & Toutes & $\boldsymbol{p} \boldsymbol{v} \leq \mathbf{0 , 1}$ & $\boldsymbol{p} \boldsymbol{v} \leq \mathbf{0 , 0 5}$ & $\boldsymbol{p} \boldsymbol{v} \leq \mathbf{0 , 0 1}$ \\
\hline 1. Méthodes des votes & & & & \\
Nombre de résultats avec un lien positif & 179 & 83 & 75 & 45 \\
Nombre total de résultats & 278 & 121 & 110 & 64 \\
Test du signe (proportion de liens positifs) & 0,64 & 0,69 & 0,68 & 0,70 \\
Probabilité d'obtenir la proportion $p$ sous $\mathrm{H}_{0}$ & $p<0,0001^{* * *}$ & $p<0,0001^{* * *}$ & $p<0,0001^{* * *}$ & $p<0,0004^{* * *}$ \\
\hline 2. Combinaison des p-values & & & & \\
Méthode des p moyens (statistique de test) & 17,76 & 18,26 & 17,63 & 13,77 \\
p cumulé (degré de signification) & $p<0,0001^{* * *}$ & $p<0,0001^{* * *}$ & $p<0,0001^{* * * *}$ & $p<0,0001^{* * *}$ \\
\hline
\end{tabular}

Note : *** indique que le résultat est significatif pour une erreur de première espèce de $1 \%$, l'hypothèse nulle est alors rejetée. Pour la méthode des votes, l'hypothèse nulle est l'absence de prépondérance des résultats positifs et significatifs. Pour la méthode de combinaison des $p$-values, il s'agit d'une hypothèse nulle omnibus : il n'existe aucun résultat significatif. Le nombre total de résultats (278) dans ce tableau est supérieur au nombre d'articles compilés dans notre méta-analyse (108), car certains articles présentent plusieurs résultats de l'étude du lien entre participation budgétaire et performance managériale (plusieurs échantillons, plusieurs modèles de tests, entre autres).

Ce tableau classe en colonne nos résultats en fonction du degré de signification ( $p$-value, notée $p v)$ du résultat des études incluses. Nous avons obtenu ces résultats en incluant soit toutes les études sur le sujet ( $2^{\mathrm{e}}$ colonne), soit seulement celles dont le lien entre participation et performance présente une $p$-value inférieure à $10 \%, 5 \%$ et enfin $1 \%$ (respectivement, $3^{\mathrm{e}}, 4^{\mathrm{e}}$ et $5^{\mathrm{e}}$ colonnes). Cela permet de dépasser, en partie, le dilemme entre pertinence des résultats et exhaustivité des méta-analyses non quantitatives.

\subsubsection{RÉSULTATS DE LA MÉTHODE DES VOTES (PARTIE 1 DU TABLEAU 1)}

Que l'on retienne l'ensemble des études disponibles («Toutes») ou seulement celles ayant des résultats avec des degrés de signification faibles ( $\langle p v \leq 0,1 », 《 p v \leq 0,05 »$ ou $« p v \leq 0,01 »)$, on peut conclure à l'existence d'un lien significativement positif entre la participation budgétaire et la performance managériale $(p<0,01)$. L'hypothèse d'absence de prépondérance des résultats positifs étudiant la relation participation budgétaire - performance managériale est rejetée. Ce résultat est, de plus, robuste à notre critère d'inclusion des études basé sur leurs $p$-values. Pour une erreur de première espèce de $1 \%$, que l'on prenne en compte uniquement les études très significatives ou toutes les études, la méthode des votes indique qu'il y a un lien significativement positif entre la participation budgétaire et la performance managériale $(p<0,01)$.

\subsubsection{RÉSULTATS DE LA COMBINAISON DES DEGRÉS DE SIGNIFICATION (PARTIE 2 DU TABLEAU 1)}

Les résultats d'une méthode de combinaison des degrés de signification permettent de confirmer les résultats univoques de la méthode des votes. Quel que soit le critère d'inclusion des études retenu, le test basé sur la méthode de la moyenne des p-values rejette l'hypothèse nulle omnibus (absence d'un effet significatif). Parmi les études empiriques sur le lien entre participation budgétaire et performance managériale, il y a au moins un résultat significatif. 


\subsection{Limites des méthodes de méta-analyse non quantitatives}

Malgré l'avantage de l'exhaustivité, les techniques de méta-analyse non quantitative qui viennent d'être présentées ont été jugées peu pertinentes pour la constitution de méta-analyse par les participants au forum de Potsdam de 1994. Premièrement, elles agrègent des résultats hétérogènes (Feinstein 1995). Deuxièmement, elles combinent des résultats (ou mesures) et non des études et retiennent souvent comme filtre le degré de signification de chaque résultat. Ainsi, Slavin (1995) soutient qu'elles donnent trop de poids aux études utilisant plusieurs échelles pour mesurer une seule variable et ayant un grand nombre de répondants.

Par ailleurs, ces techniques ne donnent pas une estimation globale de la taille de l'effet ${ }^{7}$ d'une $^{\prime}$ variable sur une autre, contrairement à une méta-analyse quantitative. Autrement dit, bien qu'elles permettent de synthétiser tous les résultats quantitatifs de la littérature, elles ne permettent pas de quantifier l'effet d'une variable sur une autre contrairement aux méta-analyses quantitatives.

Plus précisément, la combinaison des degrés de signification est un critère peu discriminant. Elle ne s'intéresse qu'à l'existence d'au moins un effet significatif sur l'ensemble des études. Elle ne permet donc pas de conclure à l'existence d'un lien global significativement positif ou négatif. Concernant la méthode des votes, Slavin (1995) considère qu'elle est souvent peu pertinente : cette technique ne prend pas en compte la qualité des articles et ne différencie pas les effets importants des effets faibles. De plus, la méthode des votes n'offre qu'une réponse concernant le signe de l'effet de la participation budgétaire sur la performance managériale, sans donner une estimation de cet effet. Seules les métaanalyses quantitatives permettent d'obtenir une telle estimation. Leur mise en œuvre et leurs résultats sont présentés dans la section suivante.

\section{Mise en ouvre d'une méta-analyse quantitative exhaustive}

Pour dépasser les limites des méta-analyses non quantitatives et des revues de littérature, un autre principe peut être appliqué : celui de la quantification de l'effet étudié. Pour respecter ce principe, l'utilisation des méta-analyses quantitatives semble intéressante.

\subsection{Deuxième principe de la méta-analyse : quantification de l'effet d'une variable sur une autre}

Un deuxième principe permet de différencier les revues de littérature classiques, les méta-analyses non quantitatives et les méta-analyses quantitatives. Les revues de littérature classiques ne présentent « pour la plupart, qu'une opinion «d'expert » plus ou moins argumentée avec quelques faits issus des [études]. Ainsi, ces articles ne constituaient pas vraiment des synthèses objectives et fiables des observations scientifiques accumulées au fil des essais » (Cucherat et al. 1997, p. 7). Une métaanalyse non quantitative permet alors de synthétiser toutes les études de façon plus systématique, quelles que soient leurs méthodes statistiques utilisées. Mais ce type de méta-analyses ne quantifie pas l'effet d'une variable sur une autre, contrairement aux méta-analyses quantitatives.

Les méta-analyses quantitatives ont pour objectif d'estimer l'effet d'une variable sur une autre en 
s'appuyant sur les effets estimés dans chacune des études prises en compte. Elles peuvent inclure toutes les études dont les résultats statistiques se prêtent à ce type de méta-analyses. Les méta-analyses quantitatives ne peuvent être mobilisées que pour synthétiser des résultats issus d'études utilisant des régressions pour estimer le lien entre deux variables. Nous détaillons les méthodes utilisées, les résultats obtenus et leurs limites dans les développements suivants.

\subsection{Méthodes des méta-analyses quantitatives}

Suivant Cucherat et al. (1997), nous mettons en œuvre différentes méthodes de méta-analyse quantitative prenant en compte tous les articles dont les résultats statistiques sont utilisables. Dans ces méta-analyses quantitatives, nous excluons notamment les études de Milani (1975) et de Tiller (1983), car celles-ci ne font pas apparaître la pente de la relation entre participation budgétaire et performance managériale, ni son écart-type. Pour voir la sensibilité des résultats à la pondération retenue, nous avons réalisé deux méta-analyses quantitatives : une avec pondération du résultat de chaque étude par un score de qualité et une autre sans pondération.

\subsubsection{PONDÉRATION DES RÉSULTATS AVEC UN SCORE DE QUALITÉ}

Pour pondérer les articles en fonction de leur qualité, Slavin (1995, p. 13) considère qu'il faut prendre en considération leurs validités interne et externe, et la pertinence des échelles de mesures utilisées.

Pour privilégier les études ayant une certaine validité interne, Slavin (1995, p. 13) conseille de juger le « canevas » de l'étude (study design) pour s'assurer que les auteurs ont tenté de minimiser les biais. Slavin souligne aussi que l'on doit s'assurer de la validité externe. Pour ce faire, il indique qu'il est particulièrement important d'écarter toute expérimentation faite sur des étudiants en nombre limité. Cette préconisation a été suivie pour cette méta-analyse, car nous étudions le lien entre la participation budgétaire et la performance des managers (et non des étudiants). Enfin et surtout, «le plus important principe d'inclusion doit être la pertinence par rapport au sujet d'étude » (Slavin 1995, p. 13). Il faut donc que les définitions des concepts retenues par chaque article inclus dans la métaanalyse soient exactement les mêmes, et que les échelles utilisées pour mesurer une variable soient identiques et non pas proches ou reliées. Cela permet d'assurer une homogénéité des mesures et une «bonne » validité conceptuelle des études incorporées.

Plus précisément, suivant Slavin (1995), les articles utilisant la méthode expérimentale sur des étudiants ont ainsi été exclus car leur validité externe est faible, puisque nous nous intéressons au lien entre participation budgétaire et performance chez les managers.

À partir de cette première sélection, une note de qualité a été attribuée à chaque article selon les critères suivants (Slavin 1995).

Premièrement, la qualité de l'explication des méthodes utilisées a été évaluée. Un article ne décrivant pas avec suffisamment de précisions la constitution de son échantillon, sa collecte de données et leur traitement, se voit attribuer un score plus faible qu'un article les décrivant plus précisément. Les notes vont de 0,8 à 1,4 sur notre échantillon d'articles ${ }^{8}$.

Deuxièmement, un critère a été fixé pour s'assurer de la validité externe des articles collectés étudiant le lien participation budgétaire - performance managériale. Un score de taille d'échantillon a été calculé à partir de la «valeur moyenne» de Gibbons et al. (1977) (SMR, cf. 2.2.1.). Si les 
résultats d'une étude se basent sur un échantillon de $n_{i}$ personnes alors le score associé est $n_{i} / \mathrm{SMR}$.

Troisièmement, la pertinence des échelles de mesure par rapport aux thèmes étudiés a été évaluée. L'utilisation de l'échelle de Milani (1975), pour la mesure de la participation budgétaire, et celle de Mahoney et al. $(1963,1965)$, pour mesurer la performance managériale, ont été privilégiées suivant le consensus émergeant de la littérature ${ }^{9}$ et la fiabilité reconnue de ces deux échelles (Derfuss 2009). Ainsi, les études qui mesurent la participation budgétaire avec l'échelle de Milani (1975) obtiennent un score de 0,5. Si elles mesurent la participation avec une échelle s'inspirant de celle de Milani (1975), ce score est de 0,25. Dans les autres cas, un score nul est attribué. Nous avons fait de même avec l'échelle de Mahoney et al. $(1963,1965)$. La somme des deux donne un " score de pertinence » sur un.

Finalement, l'échantillon est composé de 95 études dont la présentation des statistiques permet de faire une méta-analyse quantitative. Le détail de leur score de qualité est présenté en annexe 2. Dans cette analyse, nous avons dû exclure l'article de Chong et Chong (2002a), pourtant «bon » sur le plan des critères de qualité retenus, car l'article ne traite que des liens indirects entre participation budgétaire et performance managériale. Enfin, pour les études ayant plusieurs résultats, celui issu du modèle offrant le meilleur contrôle a été retenu ${ }^{10}$.

Suite à l'attribution du score de qualité, les tailles d'effet (effect sizes) ont été calculées. Ce calcul de la taille de l'effet est cependant impossible pour certaines études compte tenu de leur présentation des résultats (Milani 1975; Shields et Young 1993; Tsui 2001; Bento et White 2006). Celles-ci ne peuvent donc être comprises dans les méta-analyses quantitatives.

Une fois un « score de qualité » établi selon les critères précédents et une sélection des meilleures études réalisée, il reste à calculer les tailles d'effet du lien de chaque étude et à les agréger.

\subsubsection{CALCUL DE LA TAILLE DE L'EFFET AVEC SCORE DE QUALITÉ}

Pour une étude $i$ estimant l'effet de la variable $\mathrm{X}$ sur la variable $\mathrm{Y}$ par un modèle linéaire $\mathrm{Y}=\hat{a}_{i}$ $\mathrm{X}+\hat{b}_{i}$, la taille d'effet est estimée par :

$$
\hat{\theta}_{i}=\frac{\hat{a}_{i}}{\hat{\sigma}\left(\hat{a}_{i}\right)}
$$

Puis, l'effet global du lien étudié est calculé de la façon suivante :

$$
\hat{\theta}=\sum_{i=1}^{i=n} q_{i} \cdot w_{i} \cdot \hat{\theta}_{i} / \sum_{i=1}^{i=n} q_{i} \cdot w_{i}
$$

avec $q_{i}$ le score de qualité défini ci-dessus pour l'étude $i, w_{i}$ l'inverse de la variance de l'estimateur de l'effet de l'étude $i$ (les résultats statistiques ayant une forte variance sont faiblement pondérés).

Pour savoir si l'effet global trouvé est significatif, il faut calculer sa variance :

$$
\mathrm{V}(\hat{\theta})=\sum_{i=1}^{i=n} q_{i}^{2} \cdot w_{i} /\left(\sum_{i=1}^{i=n} q_{i} \cdot w_{i}\right)^{2}
$$


On calcule ensuite la statistique de test $\mathrm{U}=\hat{\theta}^{2} / \mathrm{V}(\hat{\theta})$ qui suit une loi du $\chi^{2}$ à un degré de liberté sous l'hypothèse nulle (absence d'effet global de la participation budgétaire sur la performance managériale).

\subsubsection{CALCUL DE LA TAILLE DE L'EFFET SANS SCORE DE QUALITÉ ET SENSIBILITÉ}

Pour juger la sensibilité de ce résultat de méta-analyse quantitative sur le lien entre participation budgétaire et performance managériale, et surtout que la subjectivité inhérente au score de qualité ne «biaise » pas la statistique, nous avons également calculé la statistique U sans le score de qualité. Cela permet de contrôler l'impact de l'utilisation d'un score de qualité : un élément pouvant biaiser la méta-analyse dans un sens ou dans l'autre. Avant de présenter les statistiques sans score de qualité, notons que les auteurs de deux des méta-analyses précédentes sur le lien entre participation budgétaire et performance managériale (Derfuss 2009; Greenberg et al. 1994) avaient utilisé comme score de qualité la taille de l'échantillon, mais ils n'avaient pas regardé l'incidence de ce choix sur le résultat de la méta-analyse.

Sans le score de qualité, nous avons les relations statistiques suivantes :

$$
\hat{\theta}=\sum_{i=1}^{i=n} \hat{\theta}_{i} \cdot w_{i} / \sum_{i=1}^{i=n} w_{i} \text { et } \mathrm{V}(\hat{\theta})=1 / \sum_{i=1}^{i=n} w_{i}
$$

La statistique de test est alors : $\mathrm{U}=\left(\sum_{i=1}^{i=n} \hat{\theta}_{i} \cdot w_{i}\right)^{2} / \sum_{i=1}^{i=n} w_{i} \stackrel{\mathcal{L}}{\rightarrow} \chi^{2}(1)$

Cette comparaison des statistiques de test avec et sans le score de qualité permet de juger l'impact du score de qualité sur le résultat final de la méta-analyse. Dans la réalisation d'un compte rendu d'une méta-analyse, il est ainsi souhaitable de retrouver cette comparaison pour que les résultats des métaanalyses soient commentés avec précaution.

\subsection{Résultats des méta-analyses quantitatives}

L'hypothèse de nullité de la taille d'effet ${ }^{11}$ a été testée, d'abord en tenant compte du score de qualité des articles, puis sans tenir compte de celui-ci afin de contrôler son impact sur les résultats et de le discuter.

Le tableau 2 présente le résultat de la méta-analyse quantitative avec et sans score de qualité.

Tableau 2

Méta-analyse quantitative

\begin{tabular}{|l|c|c|}
\hline \multicolumn{1}{|c|}{$\boldsymbol{n}=\mathbf{9 5}$} & Avec score de qualité & Sans score de qualité \\
\hline$\hat{\theta}$ (effet commun) & $-0,008$ & $0,132^{* * *}$ \\
\hline Var $(\hat{\theta})$ & 0,001 & 0,001 \\
\hline Statistique de test & 0,118 & 31,501 \\
\hline
\end{tabular}




\begin{tabular}{|l|c|c|}
\hline Fractile $\boldsymbol{\chi}^{\mathbf{2}}$ pour $\boldsymbol{\alpha}=\mathbf{1} \%$ & 6,635 & 6,635 \\
\hline $\boldsymbol{p}$-value & 0,731 & $\mathrm{p}<0,0001$ \\
\hline Résultat du test & Effet négatif non significatif & Effet positif significatif \\
\hline
\end{tabular}

Note : *** indique que le résultat est significatif pour une erreur de première espèce de $1 \%$.

Les résultats de la méta-analyse quantitative comprenant toutes les études utilisables sont mitigés, tant au niveau du signe de l'effet que de son degré de signification. Sans score de qualité, le lien entre la participation budgétaire et la performance managériale est positif et très significatif. Avec le score de qualité, ce lien devient négatif et non significatif.

\subsection{Limites d'une méta-analyse quantitative exhaustive}

Sur la base de cette méta-analyse quantitative intégrant toutes les études, il est donc difficile de conclure. C'est la principale limite de l'application des techniques de méta-analyse quantitatives au lien entre participation budgétaire et performance managériale. Comment peut-on expliquer la sensibilité de la méta-analyse quantitative au score de qualité ? Il est possible suivant la littérature d'avancer une première réponse: la volonté d'inclure toutes les études (cf. 2.1., principe d'exhaustivité) amène à prendre en compte des observations de qualité moindre.

Avant de réaliser une méta-analyse, il faut s'assurer de ne pas faire de l'«alchimie » en transformant $\mathrm{du}$ "rien en quelque chose », " du plomb en or » ou pire en travaillant avec des substances hétérogènes (Feinstein 1995, p. 71). Feinstein met en garde contre l'application de la métaanalyse sur des observations mal conçues, non « randomisées » ou hétérogènes. Au mieux, dit-il, nous risquons de nous retrouver avec une "salade composée» en mélangeant des "pommes et des oranges », au pire avec des «substances moins savoureuses ». Pour Feinstein, il faut respecter le principe scientifique d'homogénéité.

Pour évaluer l'hétérogénéité pouvant affecter les résultats d'une méta-analyse, il est possible d'utiliser une statistique spécifique : le $\mathrm{I}^{2}$. Cette statistique rend compte du pourcentage de variations des résultats entre les études expliquées par la présence d'hétérogénéité. Plus cette statistique est faible, plus l'hétérogénéité l'est aussi et plus fiable est le résultat de la méta-analyse. Higgins et al. (2003) affirment que cette statistique est plus puissante, lorsque le nombre d'études est faible. Le détail de ce test est développé dans Higgins et Thompson (2002).

Appliquée aux articles utilisés pour réaliser une méta-analyse quantitative exhaustive, cette statistique de test donne les résultats suivants. Le pourcentage de variations des résultats venant de l'hétérogénéité est de plus de 83,52\%. On peut rejeter l'hypothèse d'homogénéité, puisque la borne inférieure de l'intervalle de confiance autour de $\mathrm{I}^{2}$ est supérieure à 0 : elle est égale à 79,26\% pour une erreur de première espèce de $1 \%$.

Face à des articles peu homogènes, il est souhaitable d'oublier le premier principe (exhaustivité) et de réaliser une méta-analyse quantitative uniquement sur les «meilleures études » en appliquant le troisième principe de réalisation d'une méta-analyse : la sélection argumentée des études. Il s'agit d'une méthode pertinente d'après les conclusions du forum de Potsdam sur la méta-analyse (Feinstein 1995 ; Slavin 1995). 


\section{Mise en ouvre d'une méta-analyse réduite aux articles de qualité}

Dans la section précédente, il est apparu que la réalisation d'une méta-analyse quantitative sur l'intégralité des études pouvant faire l'objet de ce traitement pose des questions de sensibilité des résultats au score de qualité. L'hétérogénéité des études pouvant expliquer cela (cf. 3.4.), nous remplaçons ici le principe d'exhaustivité par un principe de sélection argumentée des études. Cela permet de mettre en œuvre une méta-analyse limitée aux études dites « de qualité ».

\subsection{Troisième principe de la méta-analyse : sélection argumentée des études}

La recherche d'exhaustivité amène parfois à inclure des études dégradant la qualité des méta-analyses. Par exemple, des études dont l'échantillon n'est pas sélectionné par tirage aléatoire peuvent être incluses, alors qu'elles comportent un biais d'échantillonnage. Ce biais diminue la validité externe, la possibilité de généraliser une relation observée à une population d'individus (Calder et al. 1982, p. 240). Ainsi, pour réaliser des méta-analyses de qualité, certains auteurs avancent qu'il faudrait améliorer ex ante la conception des études de terrain (Feinstein 1995, p. 77). Mais la question des critères de validité externe d'une étude empirique n'est pas simple à traiter. Les méta-analyses doiventelles privilégier des études de terrain avec de grands échantillons? Pour limiter les biais d'échantillonnage, ne doit-on pas réaliser des méta-analyses uniquement sur des études dont les échantillons ont été tirés aléatoirement? Malgré les biais de publication possibles, ne doit-on intégrer que des articles publiés dans des revues de très haut niveau académique devant assurer un certain niveau de qualité des études incorporées?

Une réponse à ces questions a été proposée par Slavin (1995). Il préconise de ne retenir que les meilleures études pour faire une méta-analyse quantitative. Cet auteur appelle cela une « synthèse des meilleures études » (best evidence synthesis). Le problème de la définition des critères caractérisant les « meilleures études » se pose alors.

\subsection{Critères de sélection et jugements de valeurs}

Ainsi, nous dépassons l'impératif d'exhaustivité en préférant n'inclure que les articles de «qualité exceptionnelle » dans le cadre d'une synthèse des meilleures études: un type de méta-analyse quantitative ne synthétisant que les études remplissant des critères de qualité fixés a priori. Pour la sélection de ces articles, nous avons suivi les recommandations de Slavin (1995) et celles des communications du forum de Potsdam de 1994. Définir des critères de sélection et d'évaluation est un processus subjectif. Ainsi, après présentation des critères, nous détaillons nos «jugements de valeurs » (Myrdal 1958).

\subsubsection{CRITÈRES DE DÉFINITION ET DE SÉLECTION DES «MEILLEURES ÉTUDES »}

Nous pouvons justifier notre sélection des articles en reprenant le résultat de la méta-analyse quantitative réalisée par Derfuss (2009). Dans cette méta-analyse, les résultats présentent des hétérogénéités concernant le lien entre participation budgétaire et performance managériale. Si l'on suit Feinstein (1995), une explication possible de ce type d'hétérogénéité est la présence, au sein de l'échantillon d'articles, d'études de «qualité moindre » au regard des critères évoqués à la section 
3.2.1., et cela même si Derfuss a réalisé sa méta-analyse sur des articles publiés dans des revues anglosaxonnes, majoritairement classées.

Si les études à synthétiser ne sont pas homogènes, une solution envisageable est de sélectionner les études en fonction de leur « qualité ». Cela permet de ne faire une méta-analyse qu'avec des études de qualité comparable et donc quasi-homogènes, sur le plan de la méthode. Il s'agira alors de ne retenir que celles qui semblent "exceptionnelles » en partant du principe que "cette sélectivité semble bien plus attractive que la combinaison de fruits, de légumes ou d'autres choses dans une méta-analyse standard qui s'abstient même des précautions scientifiques offertes par la sélection aléatoire de l'échantillon » (Feinstein 1995, p. 77, T.D.A.). Ce principe justifie l'usage d'un type de méta-analyse quantitative appelée best evidence synthesis.

Pour l'application de cette méthode, suivant Derfuss (2009) ${ }^{12}$, nous avons d'abord identifié les articles dont l'échantillon est constitué par tirage aléatoire, car cet élément renforce la validité et la robustesse des résultats obtenus dans ces études (Kendall et al. 1987 ; Feinstein 1995; Yin 2003, p. 8). Ensuite, parmi ces articles sélectionnant aléatoirement leur échantillon, nous n'avons conservé que les études ayant utilisé les échelles de mesure les plus fiables : celles de Milani (1975) pour la participation budgétaire, et celle de Mahoney et al. $(1963,1965)$ pour la performance managériale. Ainsi, notre synthèse des meilleures études a été réalisée sur les 27 études avec un dièse accolé au score de « pertinence des échelles » dans le tableau de l'annexe 2.

Même si nos critères de sélection sont justifiés et semblent objectifs, nous ne pouvons pas nous extraire de la subjectivité inhérente à toute procédure de choix et d'évaluation (comme celle du score de qualité). Ainsi, suivant Myrdal (1958), la section suivante expose nos jugements de valeur et discute de leurs effets possibles sur les résultats.

\subsubsection{JUGEMENTS DE VALEUR DES AUTEURS ET SUBJECTIVITÉ DU SCORE DE QUALITÉ}

Selon Myrdal, le chercheur arrive sur un terrain avec une idée de ce qu'il devra probablement trouver ou de ce qu'il veut trouver. Ainsi, notre travail de recherche part d'une conception opposée à celle de Max Weber qui a des prescriptions en faveur d'une science sociale totalement libérée des valeurs. Comme Myrdal (1958), nous pensons que les valeurs ont du poids. Il semble ainsi souhaitable d'exposer nos jugements de valeur pour tenter de remédier à l'impossible coupure entre énoncés de faits et énoncés de valeurs.

«La reconnaissance que nos concepts sont chargés de valeurs implique qu'ils ne sauraient se définir qu'en termes d'appréciations politiques. » (Myrdal 1958, p. 476.) Suivant Myrdal, il serait souhaitable «dans une étude [...] de clarifier et d'expliciter les préliminaires qui ont formé les jugements de valeur cachés qui pourraient conduire à des déformations de notre analyse » (Myrdal 1976, p. 36, cité par Taouil 2000). Si l'on ne fait pas cela, notre attitude « équivaut à une fraude même si elle est inconsciente » (Myrdal 1958, p. 476). Ainsi, dans le paragraphe suivant, nos jugements de valeurs sont explicités.

Nous avons abordé ce travail en ayant la volonté de réaliser une synthèse plus large que celles proposées jusqu'à présent. Notre idée de départ était de faire une synthèse exhaustive de la littérature sur ce sujet afin de clarifier le débat. N'ayant pas réalisé d'étude préalable dans ce champ de 
recherche, nous n'avions pas la volonté a priori de montrer que le lien entre participation budgétaire et performance managériale prenait un signe particulier. Suivant Derfuss (2009), nous pensions, cependant, qu'il pouvait exister des contingences expliquant les différences entre les résultats observées par les études sur ce sujet. Ce dernier jugement de valeur (en faveur d'une relation contingente) doit être pris en compte par le lecteur, car il a pu orienter in fine notre analyse. Toutefois, cet a priori, affectant l'analyse (ex post) de nos résultats, n'a pas dû avoir d'incidence significative sur les critères de sélection retenus (ex ante), issus de la littérature.

Au final, c'est dans la construction d'un score de qualité des articles que notre subjectivité a probablement eu le plus d'influence. Le choix des critères de notation, bien que justifié par la littérature, et leur application, aboutissant au score de qualité d'un article (cf. annexe 2), peuvent amener un résultat plutôt subjectif. Néanmoins pour limiter les biais, comme expliqué en note 8 et dans la note de l'annexe 2, deux des auteurs se sont penchés sur l'évaluation des articles de façon indépendante (notamment pour le critère " précision du design », le plus empreint de subjectivité) et ont ensuite confronté leur évaluation jusqu'à être d'accord sur la valeur du score. Malgré ces précautions et puisque la subjectivité du score de qualité est inhérente à sa construction, nous avons parallèlement effectué les analyses sans tenir compte de celui-ci (comme expliqué en section 3.2.2.). De cette façon, les résultats de l'étude sont reproductibles par d'autres chercheurs (qui peuvent construire leur propre score de qualité et le comparer au nôtre en même temps que reproduire le résultat de la méta-analyse sans score de qualité).

Ces précisions apportées, les lecteurs disposent des éléments pour apprécier la subjectivité attachée aux résultats de notre synthèse des meilleures études.

\subsection{Résultats des méta-analyses quantitatives des « meilleures études »}

L'hypothèse de nullité de la taille d'effet a été testée, d'abord en tenant compte du score de qualité des articles, puis sans tenir compte de celui-ci, afin de contrôler son impact sur les résultats et de le discuter. Par ailleurs, pour contrôler la sensibilité des résultats aux critères d'inclusion, les mêmes analyses ont été réalisées en ne retenant d'abord que les études basées sur un échantillon aléatoire (sans contraintes relatives à l'utilisation des échelles) et en ne retenant ensuite que les articles publiés dans des revues classées (derniers classements du CNRS et de l'AERES connus, ceux de respectivement 2008 et 2010) et la série des Advances in ${ }^{13}$, à l'instar de Derfuss (2009).

Le tableau 3 présente les résultats de la synthèse des meilleures études n'incluant que les études se basant sur des échantillons aléatoires et n'utilisant que les échelles de mesure de Milani (1975) et Mahoney et al. $(1963,1965)$. La note du tableau 3 donne les résultats du contrôle de sensibilité des synthèses des meilleures études. 


\section{Tableau 3}

Synthèse des meilleures études - Critère d'inclusion : échantillons aléatoires et échelles de mesure Milani (1975) / Mahoney et al. $(1963,1965)$

\begin{tabular}{|l|c|c|}
\hline \multicolumn{1}{|c|}{$\boldsymbol{n = 2 7}$} & Avec score de qualité & Sans score de qualité \\
\hline$\hat{\theta}$ (effet commun) & 0,038 & $0,213^{* * *}$ \\
\hline Var $(\hat{\theta})$ & 0,002 & 0,002 \\
\hline Statistique de test & 0,927 & 29,322 \\
\hline Fractile $\boldsymbol{\chi}^{\mathbf{2}}$ pour $\boldsymbol{\alpha}=\mathbf{1} \%$ & 6,635 & 6,635 \\
\hline $\boldsymbol{p}$-value & 0,336 & $\mathrm{p}<0,0001$ \\
\hline Résultat du test & Effet positif non significatif & Effet positif significatif \\
\hline
\end{tabular}

Note : *** indique que le résultat est significatif pour une erreur de première espèce de $1 \%$. À titre de contrôle, nous avons fait varier les critères d'inclusion des études (comme proxies de leur qualité) sur deux autres synthèses des meilleures études. Ces critères d'inclusion sont la qualité de la sélection de l'échantillon (toutes les études basées sur un échantillon aléatoire) et la publication des études dans des revues classées. La synthèse des meilleures études incluant toutes les études ayant utilisé des échantillons aléatoires (39 études, cf. tableau de l'annexe 2) indique un lien positif et significatif à $1 \%$ entre la participation et la performance, à la fois avec le score de qualité (effet commun $=0,318, p$-value $<0,0001$ ) et sans le score de qualité (effet commun $=0,489, p$-value $<0,0001$ ). En revanche, lorsque le seul critère d'inclusion repose sur le classement de la revue par l'AERES et le CNRS ou la publication de l'article dans la série des Advances in, la synthèse des meilleures études donne des résultats non significatifs et de signe opposé en fonction de la prise en compte, ou non, du score de qualité : avec le score de qualité l'effet commun est de $-0,053$ ( $p$-value $=0,094)$ et sans le score il est de 0,032 ( $p$-value $=0,304)$, tous deux non significatifs au seuil de $1 \%$ retenu.

Les résultats de la synthèse des meilleures études sont convergents au niveau du signe. L'effet est positif et significatif à $1 \%$, sans prise en compte du score de qualité, mais positif non significatif, avec prise en compte de celui-ci. Par ailleurs, l'effet devient positif et significatif à $1 \%$ avec ou sans score de qualité lorsque le critère d'inclusion est fondé uniquement sur la qualité de l'échantillonnage (échantillon aléatoire, note de bas du tableau 3). A contrario, il est non significatif à $1 \%$ et de signe opposé en fonction du score de qualité, lorsque le critère d'inclusion est fondé sur la publication de l'étude dans une revue classée (note de bas du tableau 3).

Finalement, bien que notre score de qualité puisse rendre le lien non significatif, les deux synthèses des meilleures études basées sur les critères d'inclusion de Slavin (1995) - échelles de mesures communes ou échantillon aléatoire - pourraient amener à conclure que le lien semble positif et significatif. Cependant, lorsqu'on ne retient en plus que les études publiées dans des revues classées, le lien n'est plus significatif et son signe varie avec la prise en compte du score de qualité. Face à cette divergence de conclusions que nous tentons d'expliquer en section 6, nous privilégions ce dernier résultat (lien non significatif), car il se fonde sur des publications dans des revues classées représentatives d'une qualité académique reconnue, en plus des critères de sélection retenus.

Ces résultats sensibles au critère d'inclusion et au score de qualité peuvent venir de biais de publication ou d'une hétérogénéité parmi les articles synthétisés. Lorsqu'on regarde la provenance des études dans les revues classées par le CNRS et l'AERES et dans la série des Advances in, on remarque une prépondérance d'études en provenance d'Australie : un pays où les études sur le lien entre participation budgétaire et performance managériale aboutissent bien souvent à un effet négatif. Cela nous a conduit à faire une analyse de sous-groupes pour révéler une contingence culturelle dans la partie discussion (cf. section 6). 


\subsection{Fiabilité relative de la méta-analyse réduite aux articles de qualité}

Lorsque les résultats d'une méta-analyse ne sont pas tous convergents, selon les critères d'inclusion retenus et les scores de qualité, il faut étudier l'existence de biais de publication et d'hétérogénéité.

Un moyen d'évaluer la présence de biais de publication dans un ensemble d'articles entrant dans une méta-analyse est le «funnel plot» (Cucherat et al. 1997, p. 301) : le nuage de points d'un funnel plot représente la taille de l'échantillon en fonction de l'effet observé pour chaque étude. Il apparaît que le nuage associé à la méta-analyse quantitative est moins symétrique (figure 1 : coefficient d'asymétrie $=-4,619, p$-value $<0,00001$ ) que celui associé à la synthèse des meilleures études (figure 2 : coefficient d'asymétrie $=-1,899, p$-value $=0,00002)$. Cela amènerait à privilégier le résultat de la synthèse des meilleures études.

\section{Figure 1}

\section{«Funnel plot» associé à la méta-analyse quantitative comprenant toutes les études}

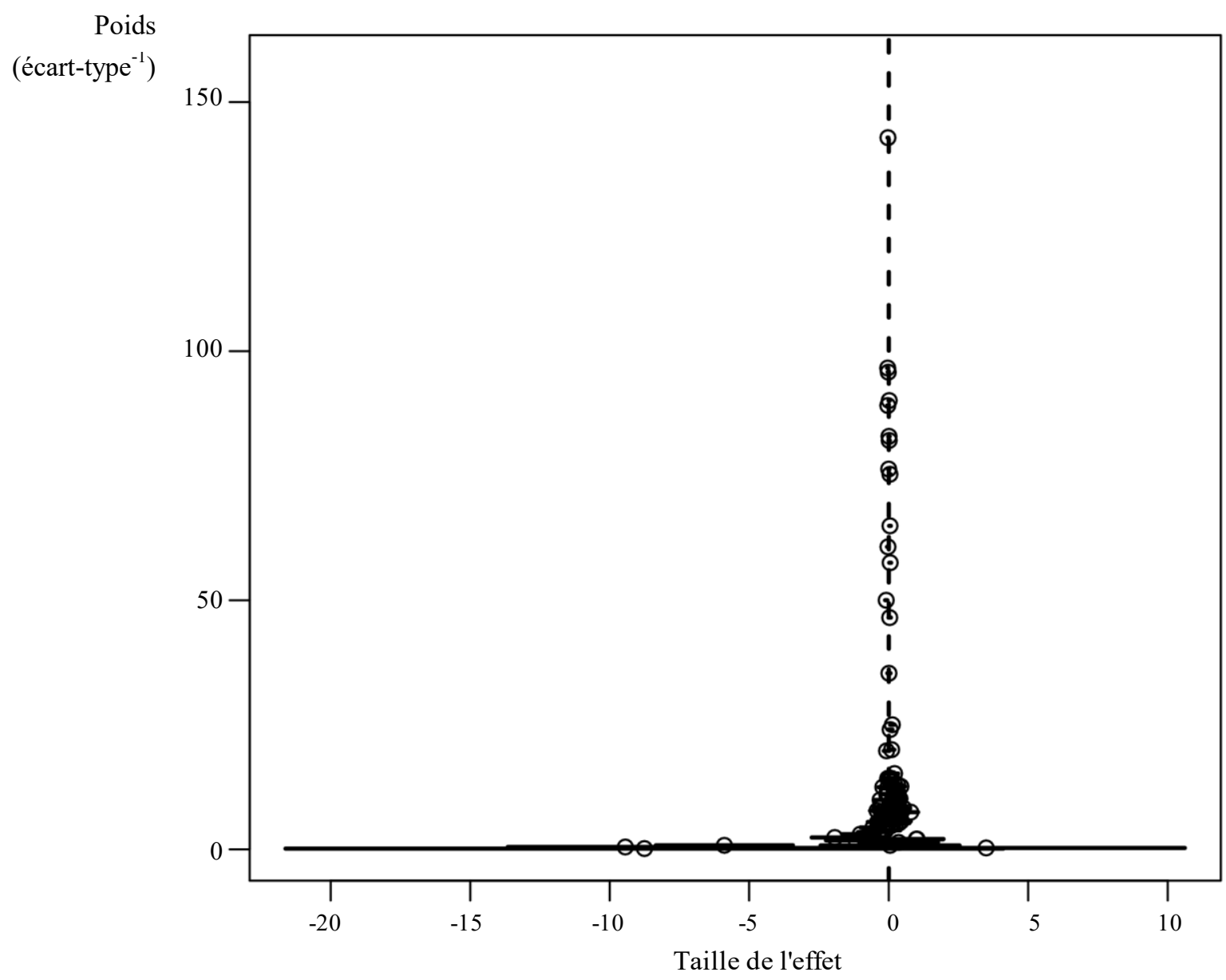

Note : Cette figure est un «funnel plot» représentant le résultat de chaque article de la méta-analyse quantitative par sa taille d'effet (en abscisses) et l'inverse de l'écart-type (en ordonnées). Un point correspond à la taille d'effet d'un article. À chaque rond est associé un trait horizontal correspondant à l'intervalle de confiance à $99 \%$ autour de la taille d'effet de ce même article. Le coefficient d'asymétrie est égal à -4,619. Un test d'asymétrie a été construit à partir de ce coefficient (Bourbonnais 2003, p. 230) dont l'hypothèse nulle est la symétrie de la distribution des tailles d'effet (c'est-à-dire que le coefficient d'asymétrie est nul). Le degré de signification est moins faible qu'avec une sélection d'articles publiés dans des revues classées ( $p$ value $<0,00001)$. Le coefficient d'asymétrie est de $-5,874$ ( $p$-value $<0,00001)$ lorsqu'on ne retient, parmi ces études, que celles publiées dans les revues « classées ». 
Figure 2

«Funnel plot » associé à la best evidence synthesis

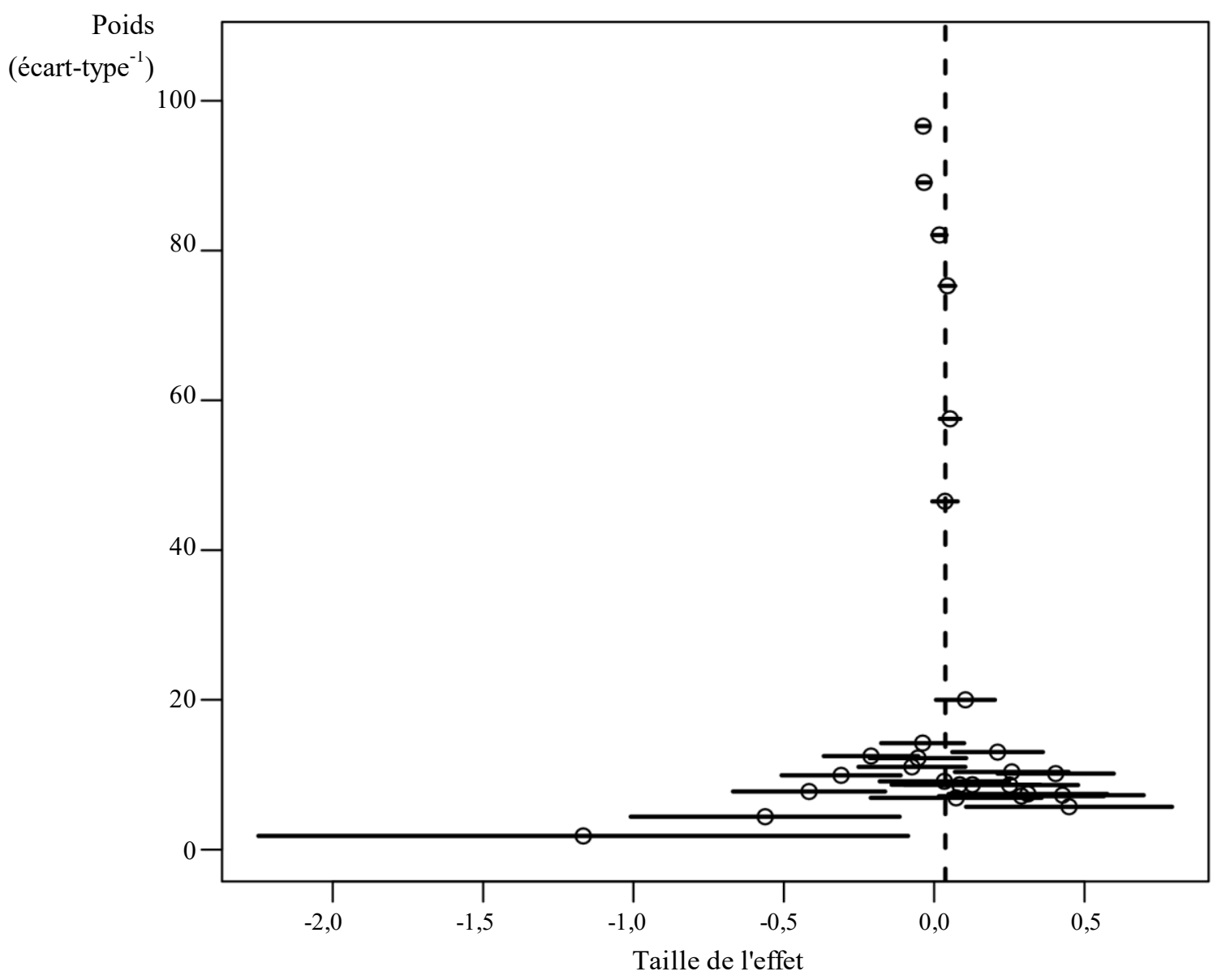

Note : Cette figure est un «funnel plot» représentant le résultat de chaque article de la méta-analyse quantitative par sa taille d'effet (en abscisses) et l'inverse de l'écart-type (en ordonnées). Un point correspond à la taille d'effet d'un article. À chaque rond est associé un trait horizontal correspondant à l'intervalle de confiance à $99 \%$ autour de la taille d'effet de ce même article. Le coefficient d'asymétrie est égal à $-1,899(p$-value $=0,00002)$. Un test d'asymétrie a été construit à partir de ce coefficient (Bourbonnais 2003, p. 230) dont l'hypothèse nulle est la symétrie de la distribution des tailles d'effet (coefficient d'asymétrie $=0$ ). Le coefficient d'asymétrie est de $-1,938$ ( $p$-value $=0,004)$ lorsqu'on ne retient, parmi ces études, que celles publiées dans les revues « classées ».

Pour vérifier si ces résultats viennent de la prise en compte d'études publiées dans des revues non classées ou non publiées, nous avons fait les mêmes tests en sélectionnant uniquement les articles publiés dans les revues classées par le CNRS et l'AERES et la série des Advances in. Dans ce dernier cas, le coefficient d'asymétrie est $-5,874$ ( $p$-value $<0,0001)$. Lorsqu'on ne prend en compte que les articles publiés dans des revues classées en France dont leur échantillon a été sélectionné aléatoirement et utilisant uniquement les échelles de Milani (1975) et Mahoney et al. (1963, 1965), le coefficient d'asymétrie est de $-1,938$ ( $p$-value $=0,004)$. On privilégiera alors le résultat de cette dernière méta-analyse.

Ainsi, que l'on ne prenne en compte que les articles publiés dans des revues classées ou non, il apparaît que les résultats issus de la méta-analyse quantitative effectuée sur les études ayant sélectionné aléatoirement leur échantillon et n'utilisant que les échelles de mesure de Milani (1975) et Mahoney et al. $(1963,1965)$ soient plus fiables, car leur distribution est plus symétrique. 
Concernant l'hétérogénéité pouvant affecter les résultats d'une méta-analyse, il est de nouveau possible d'utiliser la statistique $\mathrm{I}^{2}$ pour en rendre compte et comparer les méta-analyses quantitatives incorporant toutes les études utilisables et celles ne comprenant que les meilleures études (cf. 3.4.) Le résultat de la méta-analyse quantitative réalisée sur les meilleures études, sélectionnant aléatoirement leur échantillon et utilisant les échelles de Milani (1975) et Mahoney et al. (1963, 1965), paraît plus fiable : les études comprises sont davantage homogènes car le $\mathrm{I}^{2}$ calculé est significativement plus faible (cf. tableau 4).

\section{Tableau 4 \\ Évaluation de l'hétérogénéité}

\begin{tabular}{|c|c|c|}
\hline & Toutes les études utilisables $(\boldsymbol{n}=\mathbf{9 5})$ & Meilleures études $(\boldsymbol{n}=\mathbf{2 7})$ \\
\hline $\begin{array}{c}\mathbf{I}^{\mathbf{2}} \text { (pourcentage de la variation totale } \\
\text { due à l'hétérogénéité entre les études) }\end{array}$ & $83,52 \% * * *$ & $37,98 \% * * *$ \\
\hline Intervalle de confiance à $\mathbf{9 9} \%$ & {$[79,26 \% ; 86,91 \%]$} & {$[3,33 \% ; 60,21 \%]$} \\
\hline
\end{tabular}

Note : *** indique que le résultat est significatif pour une erreur de première espèce de $1 \%$. Des résultats similaires sont obtenus en ne retenant que les articles publiés dans des revues classées par le CNRS et l'AERES et la série des Advances in. Les bornes de l'intervalle ont été calculées en suivant la méthode de Higgins et Thompson (2002).

Cependant, l'hypothèse d'homogénéité des études synthétisées est toujours rejetée : la borne inférieure de l'intervalle autour de $\mathrm{I}^{2}$ est toujours supérieure à 0 , pour une erreur de première espèce de $1 \%$. Nous avons mené alors une analyse en sous-groupes pour expliquer cette hétérogénéité résiduelle (cf. section 6).

Avant de présenter cette analyse en sous-groupes, nous comparons les résultats obtenus avec la méta-analyse la plus fiable (celle réalisée sur les meilleures études publiées dans des revues classées, plus homogène et probablement moins affectée par un biais de publication) avec ceux des métaanalyses publiées antérieurement.

\section{Comparaison avec les précédentes méta-analyses publiées}

$\mathrm{Au}$ vu des résultats de la synthèse des meilleures études (cf. 4.3.), il semble que l'on puisse conclure qu'il n'existe pas de lien positif significatif entre participation budgétaire et performance managériale. La littérature a également montré la possibilité d'un lien négatif dans certaines circonstances : présence de contingences culturelles (Frucot et Shearon 1991; Lau et Buckland 2000 ; Bonache et al. 2010), environnementales (Chong et al. 2005 ; Affes et Boujelbene 2010) ou organisationnelles (Cherrington et Cherrington 1973 ; Brownell 1982a ; Hirst 1987 ; Mia 1988 ; Chenhall et Brownell 1988 ; Brownell et Dunk 1991; Gul et al. 1995 ; Charpentier 1998 ; Fernandez-Revuelta Perez et Robson 1999 ; Nguyen Tan Hon 2008). Ainsi, sur cette base, il pourrait être intéressant d'identifier clairement les circonstances à l'origine des relations négatives évoquées.

En caractérisant l'impact global moyen (non significatif) de la participation budgétaire sur la performance managériale, notre analyse permet de recentrer le débat scientifique sur l'étude des 
conditions (particulières) pour lesquelles cet impact global est positif significatif ou négatif significatif. Le tableau 5 résume les apports de notre étude en comparaison des précédentes métaanalyses sur le sujet. Ils sont de deux ordres : une base de données d'articles plus importante (à la fois sur le plan spatial et temporel) et l'utilisation de plusieurs méthodes de méta-analyses.

\section{Tableau 5}

\section{Comparaison de notre étude avec les précédentes synthèses}

\begin{tabular}{|c|c|c|c|c|}
\hline & Cette étude & $\begin{array}{c}\text { Bonache et al. } \\
\text { (2010) }\end{array}$ & Derfuss (2009) & $\begin{array}{c}\text { Greenberg et al. } \\
(1994)\end{array}$ \\
\hline $\begin{array}{c}\text { Sujet principal } \\
\text { traité }\end{array}$ & $\begin{array}{c}\text { Impact de la } \\
\text { participation } \\
\text { budgétaire }(\mathrm{PB}) \text { sur } \\
\text { la performance } \\
\text { managériale }(\mathrm{PM})\end{array}$ & $\begin{array}{c}\text { Impact de la } \\
\text { participation } \\
\text { budgétaire }(\mathrm{PB}) \text { sur } \\
\text { la performance } \\
\text { managériale }(\mathrm{PM})\end{array}$ & $\begin{array}{c}\text { Lien entre la } \\
\text { participation } \\
\text { budgétaire }(\mathrm{PB}) \text { et } \\
\text { les RAPM (lien entre } \\
\text { PB et PM } \\
\text { secondaire) }\end{array}$ & $\begin{array}{c}\text { Impact de la } \\
\text { participation } \\
\text { budgétaire }(\mathrm{PB}) \text { sur } \\
\text { la performance } \\
\text { managériale }(\mathrm{PM})\end{array}$ \\
\hline $\begin{array}{l}\text { Nombre d'articles } \\
\text { inclus sur le lien } \\
\text { participation - } \\
\text { performance }\end{array}$ & 108 & 15 & 28 & 32 \\
\hline $\begin{array}{l}\text { Langue(s) des } \\
\text { articles } \\
\text { synthétisés }\end{array}$ & $\begin{array}{c}\text { Anglais, chinois, } \\
\text { français, indonésien, } \\
\text { japonais }\end{array}$ & Anglais, chinois & Anglais & Anglais \\
\hline $\begin{array}{l}\text { Période couverte } \\
\text { (premier-dernier } \\
\text { article inclus) }\end{array}$ & $\begin{array}{c}1963-2010 \\
(1963-1974=2 \\
\text { articles } ; 2007- \\
2010=29 \text { articles })\end{array}$ & $1990-2007$ & $1975-2006$ & ... (inconnue $)-1992$ \\
\hline Pays couverts & $\begin{array}{l}\text { Tous les pays sur } \\
\text { lesquels des études } \\
\text { sont disponibles }\end{array}$ & $\begin{array}{c}\text { Australie, États- } \\
\text { Unis, Royaume-Uni, } \\
\text { Norvège, Singapour, } \\
\text { Taïwan }\end{array}$ & $\begin{array}{l}\text { Tous les pays sur } \\
\text { lesquels des études } \\
\text { publiées en anglais } \\
\text { dans des revues } \\
\text { classées et utilisant } \\
\text { un sondage et une } \\
\text { analyse multivariée } \\
\text { sont disponibles }\end{array}$ & $\begin{array}{c}\text { Tous les pays sur } \\
\text { lesquels des études } \\
\text { publiées en anglais et } \\
\text { utilisant un sondage } \\
\text { ou une } \\
\text { expérimentation sont } \\
\text { disponibles }\end{array}$ \\
\hline $\begin{array}{c}\text { Utilisation } \\
\text { conjointe de } \\
\text { plusieurs } \\
\text { méthodes de } \\
\text { méta-analyses }\end{array}$ & $\begin{array}{l}\text { Oui } \\
\text { (non quantitatives, } \\
\text { quantitatives et best } \\
\text { evidence synthesis) }\end{array}$ & $\begin{array}{c}\text { Non } \\
\text { (best evidence } \\
\text { synthesis) }\end{array}$ & $\begin{array}{c}\text { Oui } \\
\text { (quantitative } \\
\text { spécifique : Hunter } \\
\text { et Schmidt [2004] et } \\
\text { une forme de best } \\
\text { evidence synthesis) }\end{array}$ & $\begin{array}{c}\text { Non } \\
\text { (quantitative } \\
\text { spécifique : Hunter et } \\
\text { Schmidt }[1990] \text { ) }\end{array}$ \\
\hline Résultats & $\begin{array}{c}\text { Pas de lien positif } \\
\text { significatif selon le } \\
\text { type de méta-analyse } \\
\text { qui nous semble le } \\
\text { plus fiable dans sa } \\
\text { méthode }\end{array}$ & $\begin{array}{l}\text { Lien négatif non } \\
\text { significatif mais } \\
\text { hétérogénéité d'où } \\
\text { une analyse en sous- } \\
\text { groupes de pays : } \\
\text { - Australie : négatif }\end{array}$ & $\begin{array}{c}\text { Lien positif } \\
\text { significatif avec des } \\
\text { contingences } \\
\text { sectorielles }\end{array}$ & $\begin{array}{c}\text { Lien positif } \\
\text { significatif avec des } \\
\text { contingences suivant } \\
\text { les échelles de } \\
\text { mesure retenues }\end{array}$ \\
\hline
\end{tabular}


non significatif

- États-Unis : positif

significatif

- Taïwan : positif

significatif

À la différence des méta-analyses de Greenberg et al. (1994) et de Derfuss (2009), la nôtre met en avant un lien non significatif entre participation budgétaire et performance managériale. Partant de ce résultat, dans le point suivant, nous discutons des pistes de recherche que notre méta-analyse permet d'esquisser.

\section{Discussion}

Dans les développements suivants, nous esquissons deux pistes de recherche. Pour montrer la plausibilité de la première piste, l'existence de contingences culturelles, nous avons réalisé une analyse en sous-groupes : une méta-analyse par pays où il existe plus de deux études " de qualité ». Pour montrer la plausibilité de la seconde piste, l'existence de contingences temporelles (Bonache et al. 2010), nous avons réalisé une méta-analyse quantitative cumulative.

\subsection{Contingences culturelles : première piste de recherche}

La présence d'une hétérogénéité résiduelle dans la méta-analyse quantitative réalisée sur les meilleures études (best evidence synthesis) peut venir de la présence de contingences culturelles concernant le lien entre participation budgétaire et performance managériale (Lau et al. 1997 ; Su 2001 ; Leach-López et al. 2007 ; Leach-López et al. 2008 ; Leach-López et al. 2009 ; Bonache et al. 2010). Derfuss (2009, p. 216) avait mis en avant une étude, faite à Singapour, avec un résultat négatif (Lau et al. 1997). Mais Derfuss avait classé celle-ci comme une valeur aberrante en excluant la possibilité de contingence culturelle au motif que l'on trouvait aussi un effet négatif dans une étude faite en Australie (Mia 1988). La figure 3 montre qu'il peut exister des contingences culturelles expliquant cette hétérogénéité parmi les meilleures études. Par exemple, l'intervalle de confiance associé à une méta-analyse quantitative sur les meilleures études australiennes est disjoint de celui associé à une méta-analyse quantitative sur les meilleures études américaines. Les différences entre sous-groupes sont significatives lorsque les intervalles de confiance sont disjoints (Schmidt 1996; Viswesvaran et al. 2002). 
Figure 3

Mise en évidence de contingences culturelles

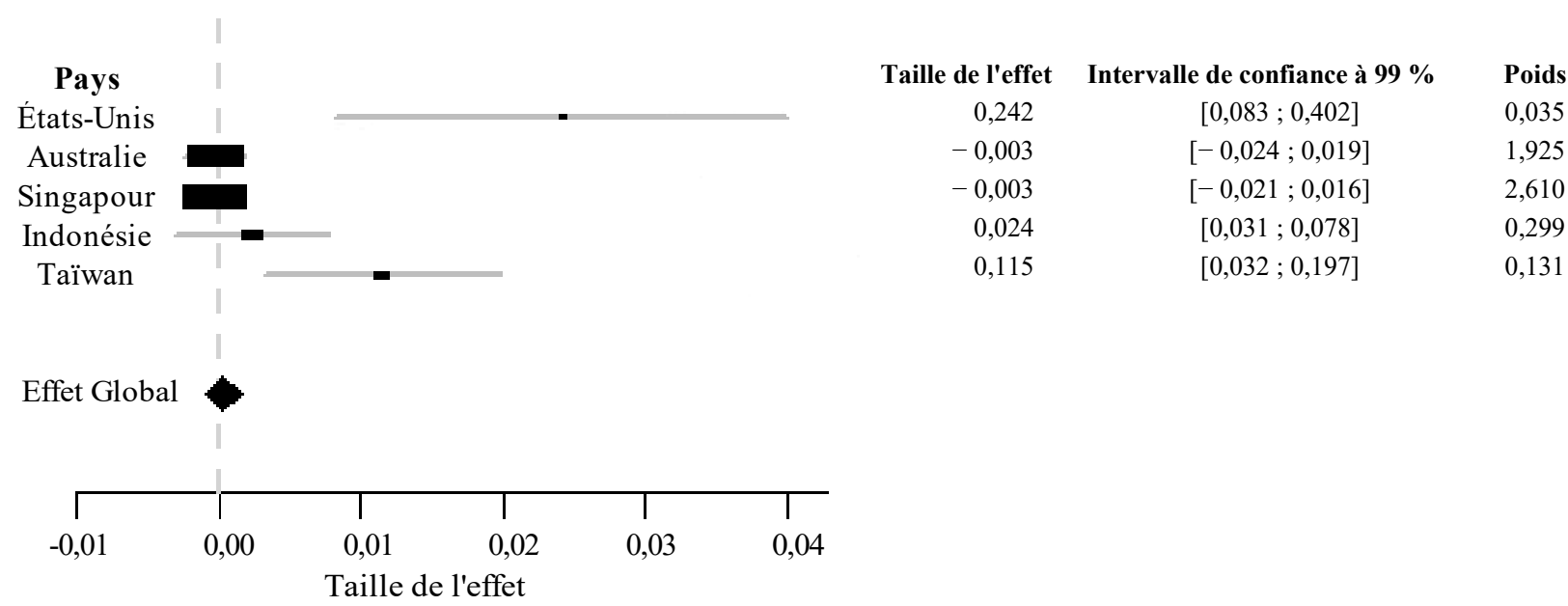

Note : Cette figure est le résultat d'une analyse en sous-groupes représentant le résultat d'une méta-analyse quantitative par pays (en ordonnées) par sa taille d'effet (en abscisses). Le milieu de chaque rectangle correspond à la taille d'effet pour un certain pays. La taille de chaque rectangle est inversement proportionnelle à la variance de la taille de l'effet. À chaque rectangle est associé un trait horizontal correspondant à l'intervalle de confiance autour de la taille d'effet pour chaque pays.

La présence de contingences, notamment culturelles, peut expliquer l'hétérogénéité des résultats parmi les études quantitatives précédentes. Dans le cadre d'une méta-analyse, l'analyse de sousgroupes permet donc d'aller dans le sens des études de cas de Charpentier (1998) et FernandezRevuleta Perez et Robson (1999) qui ont mis en avant que des contingences peuvent expliquer des résultats négatifs. Charpentier (1998) a documenté la présence d'un lien négatif entre participation budgétaire et performance managériale en situation de crise dans un service postal suédois. Fernandez-Revuelta Perez et Robson (1999) ont montré l'existence de contingences dues aux contextes politique et organisationnel, et à l'environnement organisationnel. Il pourrait alors être envisagé une étude des raisons des résultats négatifs observés en Australie et à Singapour en prenant en compte les résultats des études de cas précédentes. Cela irait dans le sens de la conclusion de la thèse de Nguyen Tan Hon (2008, p. 459), dont le travail incite « à s'interroger sur les conditions dans lesquelles se déroule la participation et semble indiquer des variables d'action, qui n'avaient pas été repérées préalablement. Les facteurs favorables à la résolution constructive des oppositions, que nous avons aussi dénommés de façon plus concise "facteurs de cohésion », doivent probablement être réunis pour pouvoir tirer tout le potentiel de la participation ».

Une fois mise en avant la possibilité de contingences culturelles pour expliquer la sensibilité des résultats à la sélection des seules études publiées dans des revues classées et la présence d'hétérogénéité entre les études, nous allons discuter d'un autre type de contingences pouvant expliquer cette hétérogénéité des résultats : les contingences temporelles.

\subsection{Contingences temporelles : seconde piste de recherche future}

L'existence possible de contingences temporelles a été avancée dans l'analyse de Bonache et al. (2010). Cette dernière ne prend en compte que les études dont l'échantillon est sélectionné 
aléatoirement dans trois pays.

Dans la méta-analyse quantitative exhaustive (celle montrant un lien positif significatif, sans score de qualité), on constate qu'il y a une prépondérance d'études récentes : 62 études datent des années 2000, contre seulement 33 avant les années 2000. Autrement dit, $35 \%$ des études seulement datent d'avant 2000 et ce pourcentage est semblable pour la méta-analyse ne retenant que les études sélectionnant aléatoirement leur échantillon et utilisant les échelles de Milani (1975) et Mahoney et al. (1963, 1965). Ces deux méta-analyses ont des résultats proches : lien positif et significatif sans score de qualité et lien non significatif avec score de qualité.

En revanche, on constate que, lorsque le critère d'inclusion est la publication dans une revue classée AERES et CNRS ou dans la série des Advances in, les études datant d'avant 2000 représentent 63,63 $\%$ des études publiées. Le résultat de la méta-analyse sur ces études publiées dans des "grandes» revues est non significatif avec ou sans score de qualité. Cela permet de supposer, comme Bonache et al. (2010), l'existence d'une contingence temporelle.

Pour montrer la plausibilité de cette proposition, nous avons réalisé une méta-analyse cumulative sur l'intégralité des études utilisables pour faire une méta-analyse quantitative. Il s'agit simplement de réaliser une méta-analyse à chaque fois qu'une nouvelle étude apparaît chronologiquement. La figure 4 représente les résultats de 95 méta-analyses itératives.

Figure 4

Méta-analyse cumulative et plausibilité de contingences temporelles

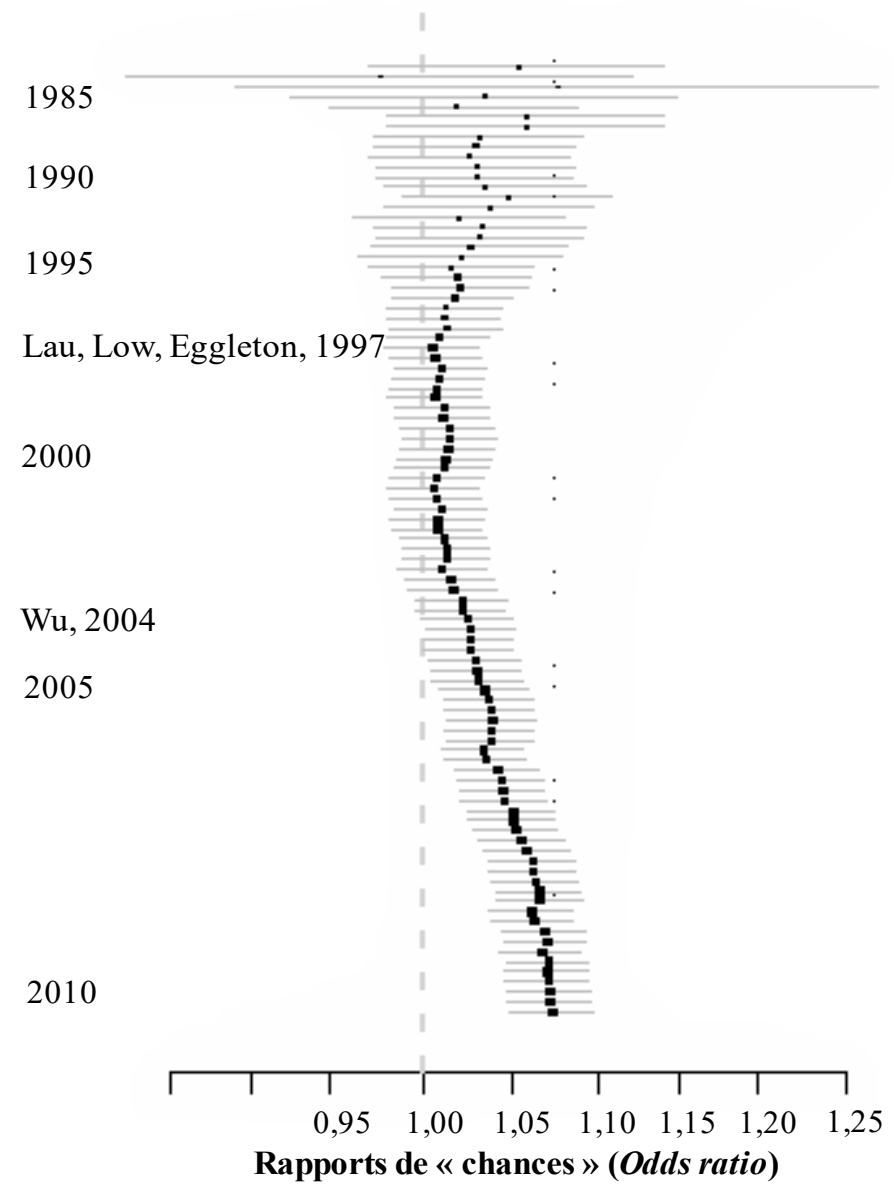


Note : Les années $1985, \ldots, 2005,2010$ ne sont pas séparées d'une même distance, car il n'y a pas eu le même nombre d'études entre ces différentes années. Le lien entre participation budgétaire et performance managériale est globalement négatif, si le rapport des « chances » de la méta-analyse cumulative est inférieur à un (à gauche de la ligne verticale en pointillés). Inversement, ce lien est positif, si ce rapport est supérieur à un (à droite de la même ligne verticale en pointillés).

On note que, avant l'étude de $\mathrm{Wu}$ (2004), les méta-analyses successives montraient un lien positif non significatif (cf. figure 4). Au-delà de cette étude, l'intervalle de confiance à $99 \%$ ne coupe plus la ligne verticale en pointillés, représentant l'absence d'effet significatif de la participation budgétaire sur la performance managériale. De plus, avant l'étude de Lau et al. (1997), on note que les métaanalyses cumulatives montraient un résultat de plus en plus faible de la participation budgétaire sur la performance managériale. Après cette étude, cette tendance semble s'être inversée.

Cette observation semble montrer que «le social n'est pas immuable ${ }^{14}$ et que le lien entre participation budgétaire et performance managériale est sujet à des contingences temporelles. On peut alors avancer l'hypothèse suivante concernant cette évolution temporelle de la méta-analyse cumulative: à force d'enseigner que la participation budgétaire augmente la performance managériale, une majorité de cadres finissent par agir dans ce sens, ce qui peut se refléter dans les études via leurs réponses aux questionnaires. Pour tester cette hypothèse, une analyse de contenu des cours enseignés et du comportement des managers pourrait être intéressante. Avec un test de Granger, il pourrait être possible de voir si les enseignements ont un effet sur les actes des managers qui auront un effet sur les résultats des études.

\section{Conclusion}

La littérature sur le lien direct entre la participation budgétaire et la performance managériale étant importante et ses résultats ambigus, cet article en réalise une synthèse exhaustive et quantifiée. Après avoir présenté un état de l'art sur le lien entre la participation budgétaire et la performance managériale et fait la synthèse de plus de cent articles ou thèses publiés sur le sujet des années 1960 à aujourd'hui, nous concluons que ce lien est globalement non significatif sur la base d'une synthèse des meilleures études. Nous montrons également que des différences culturelles et temporelles peuvent rendre ce lien significativement positif ou négatif.

Les différentes méthodes de méta-analyses mises en œuvre conduisent aux résultats suivants :

Premièrement, la combinaison des degrés de signification a permis de montrer qu'il existe au moins un résultat significatif dans la littérature. Elle ne permet toutefois pas de connaître le signe du lien entre les deux variables étudiées, d'où l'usage de la méthode des votes.

Deuxièmement, la méthode des votes a permis de montrer que ce lien est positif et significatif, mais sans en donner une estimation. Cette méthode facile à mettre en œuvre permet de renseigner le lecteur sur le degré de signification et le signe du lien sur l'ensemble de la littérature disponible, alors que les méta-analyses antérieures offraient un résultat ne s'appuyant que sur une partie des études existantes. Néanmoins, le chercheur peut avoir besoin de connaître la présence de contingences et la valeur de l'effet d'une variable sur une autre. D'où l'usage de méta-analyses quantitatives.

Troisièmement, les méta-analyses quantitatives sur toutes les études utilisables et sur les meilleures de ces études (synthèse des meilleures études) ont permis l'estimation de l'effet de la participation sur la performance et de détecter la présence et l'origine de l'hétérogénéité des résultats de la littérature. 
Cet effet est positif et significatif à $1 \%$, lorsque notre score de qualité n'est pas pris en compte et lorsqu'on retient les études basées sur des échantillons aléatoires. Le lien est non significatif, lorsqu'on utilise un score de qualité pour faire une méta-analyse quantitative et une synthèse des meilleures études (Slavin 1995), mais de signe opposé entre ces deux méthodes. Malgré le retrait d'articles n'utilisant pas une sélection aléatoire de leur échantillon, la synthèse des meilleures études comprend encore des études hétérogènes, notamment à cause de contingences culturelles. Ces contingences culturelles ont été révélées par une méta-analyse ne retenant que les articles publiés dans les revues classées par le CNRS et l'AERES ou publiées dans la série des Advances in : le résultat est non significatif et de signe ambigu en raison d'une prépondérance de travaux réalisés sur des managers australiens. Au final, nous retenons ce dernier résultat, considérant que la méthode de synthèse utilisée est la plus pertinente. La participation budgétaire ne semble donc pas avoir l'effet positif et significatif escompté sur la performance managériale au vu des études de meilleure qualité effectuées sur le sujet. Enfin, des contingences temporelles ont été révélées par une méta-analyse cumulative, indiquant que la relation est susceptible d'évoluer significativement dans le temps.

À travers cette synthèse de grande ampleur, notre article apporte un point de référence actualisé et quantifié au débat sur l'intérêt de faire participer les managers à l'élaboration de leur budget. Les résultats sont toutefois à prendre avec les précautions usuelles concernant les méthodes de synthèse : ces dernières peuvent occulter des explications contingentes importantes. Ainsi, poursuivre le débat académique sur le lien entre participation budgétaire et performance des managers autour des contingences culturelles et temporelles semble constituer une direction intéressante pour les recherches futures. 


\section{Bibliographie}

Abdullah, S.N. (1998). The role of motivation in the link between budgetary participation and managerial performance. Analisis 5 (12) : $1-17$.

Adler, R.W., Reid, J. (2008). The effects of leadership styles and budget participation on job satisfaction and job performance. Asia-Pacific Management Accounting Journal 3 (1) : 21-46.

Adrianto, Y. (2008). Analisis pengaruh partisipasi penyusunan anggaran terhadap kinerja manajerial dengan kepuasan kerja, job relevant information dan kepuasan kerja sebagai variabel moderating. Mémoire de maîtrise en comptabilité, Semarang : Université Diponegoro.

Affes, H., Boujelbene, M.A. (2010). L'impact de la participation budgétaire et de l'incertitude environnementale sur la performance et la satisfaction au travail. $31^{\mathrm{e}}$ congrès annuel de l'Association Francophone de Comptabilité, Nice.

Agbejule, A., Saarikoski, L. (2006). The effect of cost management knowledge on the relationship between budgetary participation and managerial performance. The British Accounting Review 38 : 427440.

Alam, B., Mia, L. (2006). Need for achievement, style of budgeting and managerial performance in a non government organization (NGO) : Evidence from an oriental culture. International Journal of Business Research 6 (3) : 35-43.

Alim, M.N. (2008). Efektivitas perpaduan komponen anggaran dalam prosedur anggaran : Pengujian kontinjensi matching. Jurnal Akuntansi dan Keuangan 10 (2) : 6976.

Anttila, E., Vasic, L. (2009). Delaktighet $i$ budgetprocessen inom offentlich sektor - En fallstudie inom Luleå kommun. Document de travail, Université technique de Luleå.

Aranya, N. (1990). Budget instrumentality, participation and organizational effectiveness. Journal of Management Accounting Research 2 (1) : 61-11.

Argyris, C. (1952). The impact of budgets on people. New York: Controllership Foundation.

Arifin, J. (2007). Pengaruh karakteristik gaya penyusunan anggaran terhadap efisiensi biaya. Ka Jian Bisnis Dan Manajemen 9 (1) : 23-35.

Aristote (-350). Rhetoric. Book I.

Banović, D. (2005). Evolution and critical evaluation of current budgeting practices. Mémoire de master, Ljubljana : Université de Ljubljana.

Bass, B.M., Leavitt, H.J. (1963). Some experiments in planning and operating. Management Science 9 (4) : 574-585.

Becker, B.J. (1994). Combining significance levels. In The handbook of research syntheis (Eds, Cooper, H., Hedges, L.V.). New York: Russel Sage Foundation, chapitre 15, 215-230.

Becker, S.W., Green, Jr., D. (1962). Budgeting and employee behaviour. The Journal of Business 35 (4) : 392-402.

Becker, S.W., Green, Jr., D. (1964). Budgeting and employee behavior: A rejoinder to a reply. The Journal of Business 37 (2) : 203205.

Bento, A., White, L.F. (2006). Budgeting, performance, evaluation, and compensation: A performance management model. Advances in Management Accounting (15) : 51-79.

Bonache, A.B., Maurice, J., Moris, K. (2010). A best evidence synthesis on the link between budgetary participation and managerial performance. Journal of Applied Economic Sciences 5 (2) : 34-47. 
Bourbonnais, R. (2003). Économétrie. Paris Dunod.

Breaux, K.T. (2004). The effect of program commitment on the degree of participative congruence and managerial performance in a budgeting setting. Doctorat en sciences de gestion, Baton Rouge: Université Louisiana State.

Brownell, P. (1981). Participation in budgeting, locus of control and organizational effectiveness. The Accounting Review 56 (4) : 844-860.

Brownell, P. (1982a). The role of accounting data in performance evaluation, budgetary participation, and organizational effectiveness. Journal of Accounting Research 20 (1) : 12-27.

Brownell, P. (1982b). A field study examination of budgetary participation and locus of control. The Accounting Review 57 (4) : 766-777.

Brownell, P. (1983). Leadership style, budgetary participation and managerial behaviour. Accounting, Organizations and Society 8 (4) : 307-321.

Brownell, P. (1985). Budgetary systems and the control of functionally differentiated organizational activities. Journal of Accounting Research 23 (2) : 502-512.

Brownell, P., Dunk, A.S. (1991). Task uncertainty and its interaction with budgetary participation and budget emphasis : Some methodological issues and empirical investigation. Accounting, Organizations and Society 16 (8) : 693-703.

Brownell, P., Hirst, M. (1986). Reliance on accounting information, budgetary participation, and task uncertainty : Tests of a three-way interaction. Journal of Accounting Research 24 (2) : 241-249.

Brownell, P., McInnes, M. (1986). Budgetary participation, motivation, and managerial performance. The Accounting Review 61
(4) : 587-600.

Brownell, P., Merchant, K.A. (1990). The budgetary and performance influences of product standardization and manufacturing process automation. Journal of Accounting Research 28 (2) : 388-397.

Byrne, S., Damon, F. (2008). To participate or not to participate? Voice and explanation effects on performance in a multi-period budget setting. British Accounting Review 40 (3) : 207-227.

Calder, B.J., Phillips, L.W., Tybout, A.M. (1982). The concept of external validity. Journal of Consumer Research 9 (3) : 240244.

Chalos, P., Haka, S. (1989). Participative budgeting and managerial performance. Decision Sciences 20 (2) : 334-347.

Chalos, P., Poon, M. (2001). Participative budgeting and performance: A state of the art review and re-analysis. Advances in Management Accounting (10) : 171-204.

Chang, Y.-W., Chang, R.-D., Chang, C.-P., Wu, S.-C. (2007). The effect of participative budgeting congruence on the individual performance and corporate performance. The International Journal of Accounting Studies 45 (7) : 57-97.

Charpentier, C. (1998). Budgetary participation in a public service organization. Document de travail, Series in Business Administration.

Chenhall, R.H., Brownell, P. (1988). The effect of participative budgeting on job satisfaction and performance: Role ambiguity as an intervening variable. Accounting, Organizations and Society 13 (3) : 225-233.

Cherrington, D.J., Cherrington, J.O. (1973). Appropriate reinforcement contingencies in the budgeting process. Empirical Research in Accounting Selected Studies (Supplement) : 225-253. 
Chong, V.K., Bateman, D. (2000). The effect of role stress on budgetary participation and job satisfaction-performance linkages: A test of two different models. Advances in Accounting Behavioral Research (3) : 91118.

Chong, V.K., Chong, K.M. (2002a). Budget goal commitment and informational effects of budget participation on performance : A structural equation modelling approach. Behavioral Research in Accounting 14 (1) : 65-86.

Chong, V.K., Chong, K.M. (2002b). The role of feedback on the relationship between budgetary participation and performance. Pacific Accounting Review 14 (2) : 33-55.

Chong, V.K., Eggleton, I.R.C., Leong, M.K.C. (2005). The impact of market competition and budgetary participation on performance and job satisfaction: A research note. The British Accounting Review 37 : 115-133.

Chong, V.K., Eggleton, I.R.C., Leong, M.K.C. (2006). The multiple roles of participative budgeting on job performance. Advances in Accounting 22 : 67-95.

Chong, V.K., Johnson, D.M. (2007). Testing a model of antecedents and consequences of budgetary participation on job performance. Accounting and Business Research 37 (1) : 3-19.

Chow, C.W., Shields, M.D., Wu, A. (1999). The importance of national culture in the design of and preference for management controls for multi-national operations. Accounting, Organizations and Society 24 (5-6) : 441-461.

Christensen, J. (1982). The determination of performance standards and participation. Journal of Accounting Research 20 (2): 589-603.

Clinton, B.D., Hunton, J. (2001). Linking participative budgeting congruence to organization performance. Behavioral
Research in Accounting 13 : 127-142.

Cooper, H., Hedges, L. (1994). The handbook of research synthesis. Russell Sage Foundation.

Cucherat, M., Boissel, J.-P., Leizorovicz, A. (1997). Méta-analyse des essais thérapeutiques. Paris : Masson.

Damanik, M. (2009). Pengaruh anggaran partisipatif terhadap kinerja kepala sekolah dengan budget based incentive sebagai variabel intervening di Tanjung Balai. Mémoire de maîtrise en comptabilité, Medan : Université Sumatera Utara.

Damayanti, T. (2007). Pengaruh komitmen anggaran dan kultur organisasional terhadap hubungan partisipasi penganggaran dan kinerja manajerial pada kondisi stretch targets. Jurnal Akuntansi \& Auditing Indonesia 11 (1) : 81-101.

Derfuss, K. (2009). The relationship of budgetary participation and reliance on accounting performance measures with individual-level consequent variables: A meta-analysis. European Accounting Review 18 (2) : 203-239.

Din, M. (2008). Anteseden dan konsekuensi partisipasi penganggaran (Studi empiris pada satuan kerja perangkat daerah pemerintah daerah kota Palu). Mémoire de maîtrise en comptabilité, Semarang: Université Diponegoro.

Dunk, A.S. (1989). Budget emphasis, budgetary participation and managerial performance: A note. Accounting, Organizations and Society 14 (4) : 321-324.

Dunk, A.S. (1990). Budgetary participation, agreement on evaluation criteria and managerial performance: A research note. Accounting, Organizations and Society 15 (3) : 171-178.

Dunk, A.S. (1993). The effect of budget emphasis and information asymmetry on the relation between budgetary 
participation and slack. The Accounting Review 68 : 400-410.

Dunk, A.S. (1995a). The joint effect of participative budgeting and managerial interest in innovation on departmental performance. Scandinavian Journal of Management 11 (1) : 75-85.

Dunk, A.S. (1995b). Reliance on budgetary control, environmental uncertainty, and the performance of manufacturing and marketing units. Asian Review of Accounting 3 (2) : 3-15.

Eker, M. (2009). The impact of budget participation on managerial performance via organizational commitment : A study on the top 500 firms in Turkey. Ankara Üniversitesi SBF Dergisi 64 (4) : 117-136.

Etemadi, H., Dilami, Z.D., Bazaz, M.S., Parameswaran, R. (2009). Culture, management accounting and managerial performance: Focus Iran. Advances in International Accounting 25 : 216-225.

Fahrianta, R.Y. (2001). Pengaruh tidak langsung sistem penganggaran terhadap kinerja manajerial: Motivasi sebagai variabel intervening (Studi empiris pada perusahan manufaktur di Indonesia). Mémoire de maîtrise en comptabilité, Semarang : Université Diponegoro.

Feinstein, A.R. (1995). Meta-analysis : statistical alchemy for the 21 st century. Journal of Clinical Epidemiology 48 (1) : 71-79.

Fernandez-Revuelta Perez, L., Robson, K. (1999). Ritual legitimation, de-coupling and the budgetary process: Managing organizational hypocrisies in a multinational company. Management Accounting Research 10 (4) : 383-407.

Fraser, S.G. (1995). The role of commitment in the budgeting process. Document de travail, Université de Melbourne.

Frucot, V., Shearon, W.T. (1991). Budgetary participation, locus of control, and Mexican managerial performance and job satisfaction. The Accounting Review 66 (1) : 80-99.

Frucot, V., White, S. (2006). Managerial levels and the effects of budgetary participation on managers. Managerial Auditing Journal 21 (1-2) : 191-206.

Gibbons, J.D., Olkin, I., Sobel, M. (1977). Selecting and ordering populations : A new statistical methodology. Philadelphie: Wiley publication.

Godener, A., Fornerino, M. (2005). Pour une meilleure participation des managers au contrôle de gestion. Comptabilité Contrôle-Audit 11 (1) : 121-140.

Godener, A., Fornerino, M. (2009). La participation des managers au contrôle de gestion et leur performance managériale. $30^{\mathrm{e}}$ congrès annuel de l'Association Francophone de Comptabilité, Strasbourg.

Govindarajan, V. (1984). Appropriateness of accounting data in performance evaluation : An empirical examination of environmental uncertainty as an intervening variable. Accounting, Organizations and Society 9 (2) : 125-135.

Govindarajan, V. (1986). Impact of participation in the budgetary process on managerial attitudes and performance: Universalistic and contingency perspectives. Decision Sciences 17 (Fall) : 496-516.

Govindarajan, V., Gupta, A.K. (1985). Linking control systems to business unit strategy : Impact on performance. Accounting, Organizations and Society 10 (1) : 51-66.

Greenberg, P.S., Greenberg, R. H., Nouri, H. (1994). Participative budgeting: A metaanalytic examination of methodological moderators. Journal of Accounting Literature 13 : 117-141.

Grégoire, G., Derderian, F., Lelaurier, J. 
(1995). Selecting the language of the publications included in a meta-analysis : Is there a tower of Babel bias? Journal of Clinical Epidemiology 48 (1) : 159-163.

Gul, F.A., Tsui, J.S.L., Fong, S.C.C., Kwok, H.Y.L. (1995). Decentralisation as a moderating factor in the budgetary participation-performance relationship : Some Hong Kong evidence. Accounting and Business Research 25 (98) : 107-113.

Gupta, A.K., Govindarajan, V. (1984). Business unit strategy, managerial characteristics, and business unit effectiveness at strategy implementation. Academy of Management Journal 27 (1) : 25-41.

Hafiz, F.W. (2007). Pengaruh partisipasi anggaran terhadap kinerja manajerial pada PT Cakra Compact aluminium industries. Mémoire de maîtrise en comptabilité, Medan : Université Sumatera Utara.

Hassel, G.H., Cunningham, G.M. (1996). Budget effectiveness in multinational corporations : An empirical test of the use of budget controls moderated by two dimensions of budgetary participation under high and low environmental dynamism. Management International Review 36 (3) : 245-266.

Heath, R.S., Brown, Jr., J.F. (2007). A reexamination of the effect of job-relevant information on the budgetary participationjob performance relation during an age of employee empowerment. Journal of Applied Business Research 23 (1) : 111124.

Hedges, L.V., Olkin, I. (1980). Vote-counting methods in research synthesis. Psychological Bulletin 88 (2) : 359-369.

Hedges, L.V., Olkin, I. (1985). Statistical methods for meta-analysis. Orlando: Academic Press.
Hehanussa, S.J. (2001). Pengaruh locus of control terhadap hubungan antara partisipasi penyusunan anggaran dengan kepuasan kerja dan kinerja manajerial. Mémoire de maîtrise en comptabilité, Semarang : Université Diponegoro.

Heneman, H.G. (1974). Comparisons of selfand superior ratings of managerial performance. Journal of Applied Psychology 59 (5) : 638-642.

Higgins, J., Thompson, S.G. (2002). Quantifying heterogeneity in a metaanalysis. Statistics in Medicine 21 (11): 1539-1558.

Higgins, J., Thompson, S.G., Deeks, J.J., Altman, D.G. (2003). Measuring inconsistency in meta-analyses. British Medical Journal 327 (7414) : 557-560.

Hirst, M.K. (1987). Some further evidence on the effects of budget use and budget participation on managerial performance. Australian Journal of Management 12 (1) : 49-56.

Hofstede, G.H. (1967). The game of budget control. Assen : Van Gorcum.

Hoque, Z., Brosnan, P. (2007). Aligning industrial relations risk, budgetary participation, and budgeting measures of performance: Impact on managerial performance. Document de travail.

Hunter, J.E., Schmidt, F.L. (1990). Methods of meta-analysis : Correcting error and bias in research findings. Newbury Park: Sage Pub.

Hunter, J.E., Schmidt, F.L. (2004). Methods of meta-analysis: Correcting error and bias in research findings. Thousand Oaks : Sage Pub.

Iriyadi, Gurd, B. (1998). Cultural effects of budgetary participation: Indonesian evidence. Asian Review of Accounting 6 (2) : 71-100. 
Ivancevich, J.M. (1977). Different goal setting treatments and their effects on performance and job satisfaction. Academy of Management Journal 20 (3) : 406-419.

Jermias, J., Setiawan, T. (2008). The moderating effects of hierarchy and control systems on the relationship between budgetary participation and performance. International Journal of Accounting 43 (3) : 268-292.

Kang, S.H., O'Brien, J., Sivaramakrishnan, K. (1994). Analysts' interim earnings forecasts: Evidence on the forecasting process. Journal of Accounting Research 32 (1) : 103-112.

Kendall, M.G., Stuart, A., Ord, J.K. (1987). Kendall's advanced theory of statistic. Londres : Charles Griffen.

Kenis, I. (1979). Effects of budgetary goal characteristics on managerial attitudes and performance. The Accounting Review 54 (4) : 707-721.

Khalil, N.B. (2009). Task uncertainty, participative budget and performance: The case of KIC group of companies. Mémoire de master, Kuala Lumpur: Université Malaya.

Kobori, K. (2006). The effect of budgetary participation on performance: Role ambiguity and organizational commitment as an intervening variable. Mémoire de master, Tainan City: Université National Cheng Kung.

Kren, L. (1992). Budgetary participation and managerial performance: The impact of information and environmental volatility. The Accounting Review 67 (3) : 511-526.

Latif, A.B. (2007). Hubungan antara keadilan procedural dan kinerja manajerial dengan partisipasi anggaran sebagai variabel intervening (Penelitian terhadap manajer perusahaan manufaktur di Jawa tengah). Mémoire de maîtrise en comptabilité,
Semarang : Université Diponegoro.

Lau, C.M., Buckland, C. (2000). Budget emphasis, participation, task difficulty and performance : The effect of diversity within culture. Accounting and Business Research 31 (1) : 37-55.

Lau, C.M., Lim, E.W. (2002a). The intervening effects of participation on the relationship between procedural justice and managerial performance. British Accounting Review $34: 55-78$.

Lau, C.M., Lim, E.W. (2002b). The effects of procedural justice and evaluative styles on the relationship between budgetary participation and performance. Advances in International Accounting 19 : 139-160.

Lau, C.M., Low, L.C., Eggleton, I.R.C. (1995). The impact of reliance on accounting performance measures on job-related tension and managerial performance : Additional evidence. Accounting, Organizations and Society 20 (5) : 359-381.

Lau, C.M., Low, L.C., Eggleton, I.R.C. (1997). The interactive effect of budget emphasis, participation and task difficulty on managerial performance : A cross-cultural study. Accounting, Auditing and Accountability Journal 10 (2) : 175-197.

Lau, C.M., Tan, J.J. (1998). The impact of budget emphasis, participation and task difficulty on managerial performance: A cross-cultural study of the financial services sector. Management Accounting Research $9: 153-183$.

Leach-López, M.A., Stammerjohan, W.W., Lee, K.S. (2009). Budget participation and job performance of South Korean managers mediated by job satisfaction and job relevant information. Management Research News 32 (3) : 220-238.

Leach-López, M.A., Stammerjohan, W.W., McNair, F.M. (2007). Differences in the role of job-relevant information in the 
budget participation-performance relationship among US and Mexican managers: A question of culture or communication. Journal of Management Accounting Research 19 (1) : 105-136.

Leach-López, M.A., Stammerjohan, W.W., Rigsby, J.T. (2008). An update on budgetary participation, locus of control, and the effects on Mexican managerial performance and job satisfaction. Conférences 2008 European Applied Business Research (EABR) et Teaching and Learning College (TLC), San Juan.

Libby, T. (1999). The influence of voice and explanation on performance in a participative budgeting setting. Accounting, Organizations and Society 24 (2) : 125-137.

Lindquist, T.M. (1995). Fairness as an antecedent to participative budgeting: Examining the effects of distributive justice and referent cognitions on satisfaction and performance. Journal of Management Accounting Research 7 : 122-147.

Lipták, T. (1958). On the combination of independent tests. Magyar Tudomanyos Akademia Matematikai Kutato Intezetenek Kozlemenyei 3 : 171-197.

Locke, E.A., Bryan, J.F. (1967). Performance goals as determinants of level of performance and boredom. Journal of Applied Psychology 51 (2) : 120-130.

Magee, R.P. (1980). Equilibria in budget participation. Journal of Accounting Research 18 (2) : 551-573.

Mahoney, T.A., Jerdee, T.H., Carroll, S.J. (1963). Development of managerial performance: A research approach. Oxford : South-Western Publishing.

Mahoney, T.A., Jerdee, T.H., Carroll, S.J. (1965). The job(s) of management. Industrial Relations 4 (2) : 97-110.

Merchant, K.A. (1981). The design of the corporate budgeting system : Influences on managerial behavior and performance. The Accounting Review 56 (4) : 813-829.

Merchant, K.A. (1984). Influences on departmental budgeting: An empirical examination of a contingency model. Accounting, Organizations and Society 9 (3-4) : 291-307.

Merchant, K.A., Otley, D.T. (2007). A review of the literature on control and accountability. In Handbook of management accounting research (Eds, Chapman, C.S., Hopwood, A.G., Shields, M.D.). (2) : 785-802.

Mia, L. (1988). Managerial attitude, motivation and the effectiveness of budget participation. Accounting, Organizations and Society 13 (5) : 465-475.

Mia, L. (1989). The impact of participation in budgeting and job difficulty on managerial performance and work motivation: A research note. Accounting, Organizations and Society 14 (4) : 347-357.

Mia, L., Patiar, A. (2002). The interactive effect of superior-subordinate relationship and budget participation on managerial performance in the hotel industry: An explanatory study. Journal of Hospitality \& Tourism Research 26 (3) : 235-257.

Milani, K. (1975). The relationship of participation in budget-setting to industrial supervisor performance and Attitudes : A field study. The Accounting Review 50 (2) : 274-284.

Murray, D. (1990). The performance effects of participative budgeting : An integration of intervening and moderating variables. Behavioral Research in Accounting 2 (2) : 104-123.

Myrdal, G. (1958). Une économie internationale. Paris : Presses universitaires de France.

Myrdal, G. (1976). The meaning and validity of institutional economics. In Economics in 
the future (Ed, Dopfer, K.). Londres: Macmillan Press.

Nguyen Tan Hon, V. (2008). Débats et conflits lors de l'élaboration du budget. Doctorat en sciences de gestion, Paris : HEC.

Ni, F.Y., Su, J.T. (2001). The effect of organizational size and motivation on the effectiveness of participative budgeting systems. Commerce \& Management Quarterly 2 (3) : 299-318

Ni, F.Y., Su, C., Chung, S., Cheng, K. (2005). The role of trust in supervisor in budgeting system. $17^{\mathrm{e}}$ "Asia-Pacific Conference on International Accounting Issues », Wellington.

Ni, F.Y., Su, M., Su, J.T. (2003). The impact of organizational commitment on the relationship between budgetary participation and performance: A crosscultural study. $\quad 12^{\mathrm{e}} \quad$ "International Conference on Comparative Management, College of Management National Sun YatSen University », Kaohsiung.

Nor, W. (2007). Desentralisasi dan gaya kepemimpinan sebagai variabel moderating dalam hubungan antara partisipasi penyusunan anggaran dan kinerja manajerial. $10^{\mathrm{e}}$ "Simposium nasional akuntansi », Makassar.

Nouri, H., Blau, G., Shahid, A. (1995). The effect of socially desirable responding (SDR) on the relation between budgetary participation and self-reported job performance. Advances in Management Accounting 4 : 163-177.

Nouri, H., Kyj, L. (2008). The effect of performance feedback on prior budgetary participative research using survey methodology: An empirical study. Critical Perspectives on Accounting 19: 14311453.

Nouri, H., Parker, R.J. (1998). The relationship between budget participation and job performance : The roles of budget adequacy and organizational commitment. Accounting, Organizations and Society 23 (5) : 467-483.

Olkin, I. (1995). Statistical and theoretical considerations in meta-analysis. Journal of Clinical Epidemiology 48 (1) : 133-146.

Orpen, C. (1992). Job difficulty as a moderator of the effect of budgetary participation on employee performance. The Journal of Social Psychology 132 (5) : 695-696.

Otley, D., Pollanen, R.M. (2000). Budgetary criteria in performance evaluation: A critical appraisal using new evidence. Accounting, Organizations and Society 25 (4) : 483-496.

Parker, R.J., Kyj, L. (2006). Vertical information sharing in the budgeting process. Accounting, Organizations and Society 31 (1) : 27-45.

Penno, M. (1990). Accounting systems, participation in budgeting, and performance evaluation. The Accounting Review 65 (3) : 520-536.

Puspaningsih, A. (2003). Pengaruh partisipasi dalam penyusunan anggaran terhadap kepuasan kerja dan kinerja manajer : Role ambiguity sebagai variable antara. Jurnal Akuntansi \& Auditing Indonesia 7 (2) : 95116.

Puspaningsih, A. (2004). Faktor-faktor yang berpengaruh terhadap kepuasan kerja dan kinerja manajer peruhsahaan manufaktur. Jurnal Akuntansi \& Auditing Indonesia 8 (1) : 65-80.

Qi, Y. (2010). The impact of the budgeting process on performance in small and medium-sized in China. Doctorat en sciences de gestion, Twente : Université de Twente.

Quirin, J.J., Donnelly, D.P., O'Brian, D. (2000). Consequences of participative budgeting: The roles of budget-based 
compensation, commitment, and managerial performance. Advances in Management Accounting 9 : 127-143.

Quirin, J.J., Donnelly, D.P., O'Brian, D. (2004). A nomological framework of budgetary participation and performance : A structural equation analysis approach. Advances in Management Accounting 13 : 143-165.

Roethlisberger, F.J., Dickson, W.J. (1939). Management and the workers : An account of a research program conducted by Western Electric Company. Hawthorne Works, Chicago, Cambridge, Massachusetts : Harvard University Press.

Sardjito, B., Muthaher, O. (2007). Pengaruh partisipasi penyusunan anggaran terhadap kinerja aparat pemerintah daerah : budaya organisasi dan komitmen organisasi sebagai variabel moderating. $10^{\mathrm{e}}$ «Simposium nasional akuntansi », Makassar.

Schmidt, F.L. (1996). Statistical significance testing and cumulative knowledge in psychology: Implications for training of researchers. Psychological Methods 1 (2) : 115-129.

Shields, M.D., Deng, F.J., Kato, Y. (2000). The design and effects of control systems : Tests of direct- and indirect-effects models. Accounting, Organizations and Society 25 (2) : 185-202.

Shields, J.F., Shields, M.D. (1998). Antecedents of participative budgeting. Accounting, Organizations and Society 23 (1) : 49-76.

Shields, J.F., Young, S.M. (1993). Antecedents and consequences of participative budgeting: Evidence on the effects of asymmetrical information. Journal of Management Accounting Research 5 (1) : 265-280.

Slavin, R.E. (1995). Best evidence synthesis :
An intelligent alternative to meta-analysis. Journal of Clinical Epidemiology 48 (1) : 918 .

Stevanny, D.M., Merita, S.D.H. (2005). Pengaruh partisipasi manajer dalam penyusunan anggaran terhadap kepuasan kerja dan kinerja manajerial (Studi kasus pada PT. Arta Glory Buana di Sidoarjo). Mémoire de maîtrise en comptabilité, Surabaya : Université Kristen Petra.

Su, C.-C. (2008). The viewpoints of SCT and AET on the budgetary system. Doctorat en sciences de gestion, Kaohsiung : Université National Sun Yat-Sen.

$\mathrm{Su}, \mathrm{M}$. (2001). The influence of budgetary participation of the administrators of Japanese companies in Taiwan to the managerial performance: Organizational commitment as intervening variable. Mémoire de master, Kaohsiung : Université National Sun Yat-Sen.

Su, J.T., Lin, W.L. (2007). The effect of managerial performance in participative budgetary setting : Information asymmetry as an intervening variable. Document de travail.

Subramaniam, N., Ashkanasy, N.M. (2001). The effect of organisational culture perceptions on the relationship between budgetary participation and managerial jobrelated outcomes. Australian Journal of Management 26 (1) : 35-54.

Sumarno, S.E. (2006). Pengaruh system pengendalian terhadap kinerja manajerial (Studi kasus pada perusahaan pelayaran di Semarang). Mémoire de maîtrise en comptabilité, Semarang: Université Diponegoro.

Swieringa, R.J., Moncur, R.H. (1975). Some effects of participative budgeting on managerial behavior. New York : National Association of Accountants.

Syah, D.H. (2009). Pengaruh partisipasi 
penganggaran dan keadilan prosedural terhadap kinerja manajerial pada Inna Dharma Deli Medan. Mémoire de maîtrise en comptabilité, Medan: Université Sumatera Utara.

Taouil, R. (2000). Max Weber, Gunnar Myrdal et le statut de la normativité. Cahiers d'économie politique 36 : 59-70.

Taylor, D., Abdul-Hamid, F., Mohd-Sanusi, Z. (2008). The factors impacting managerial performance in local government authorities within an Islamic cultural setting. Journal of Applied Management Accounting Research 6 (2) : 73-89.

Tiller, M. (1983). The dissonance model of participative budgeting: An empirical exploration. Journal of Accounting Research 21 (2) : 581-595.

Tintri, D. (2002). Pengaruh struktur dan kultur organisasional terhadap keefektifan anggaran partisipasif dalam peningkatan kinerja managerial. Jurnal Ekonomi \& Bisnis 2 (7) : 59-68.

Tsamenyi, M., Mills, J. (2002). Perceived environmental uncertainty, organizational culture, budget participation and managerial performance in Ghana. Journal of Transnational Management Development 8 (1-2) : 17-52.

Tsui, J.S.L. (2001). The impact of culture on the relationship between budgetary participation, management accounting systems, and managerial performance : An analysis of Chinese and Western managers. International Journal of Accounting 36 (2) : 125-146.

Viswesvaran, C., Schmidt, F.L., Ones, D.S. (2002). The moderating influence of job performance dimensions on convergence of supervisory and peer ratings of job performance : Unconfounding construct-level convergence and rating difficulty. Journal of Applied Psychology
87 (2) : 345-354.

Vroom, V.H., Mann, F.C. (1960). Leader authoritarianism and employee attitudes. Personnel Psychology 13 (2) : 125-140.

Walker, K.B., Johnson, E.N. (1999). The effects of a budget-based incentive compensation scheme on the budgeting behavior of managers and subordinates. Journal of Management Accounting Research 11 : 1-28.

Welsch, G., Hilton, R., Gordon, P. (1988). Budgeting: Profit planning and control. États-Unis : Prentice Hall.

Wentzel, K. (2002). The influence of fairness perceptions and goal commitment on managers' performance in a budget setting. Behavioral Research in Accounting 14 (1) : 247-271.

Winata, L., Mia, L. (2005). Information technology and the performance effect of managers' participation in budgeting: Evidence from the hotel industry. International Journal of Hospitality Management 4 (1) : 21-39.

$\mathrm{Wu}$, S.-C. (2004). The effect of participative budgeting congruence on the individual performance and corporate performance. Mémoire de master, Taichung: Université Providence.

Yahya, M.N., Ahmad, N.N.N., Fatima, A.H. (2008). Budgetary participation and performance: Some Malaysian evidence. International Journal of Public Sector Management 21 (6) : 658-673.

Yin, R.K. (2003). Case study research : Design and methods. Thousand Oaks : Sage Pub.

Young, S.M. (1985). Participative budgeting: The effects of risk aversion and asymmetric information on budgetary slack. Journal of Accounting Research 23 (2) : 829-842.

Yuen, D.C.Y. (2007). Antecedents of budgetary participation: Enhancing 
employees' job performance. Managerial Auditing Journal 22 (5) : 533-548.

Yuen, D.C.Y., Cheung, K.C.C. (2003). Impact of participation in budgeting and information asymmetry on managerial performance in the Macau service sector. Journal of Applied Management Accounting Research 1 (2) : 65-78.

Yunita, Y. (2009). Pengaruh partisipasi penyusunan anggaran terhadap kinerja manajerial: Komitmen organisasi dan kecukupan anggaran sebagai variabel kontinjen (Studi kasus pada Universitas Dian Nuswantoro Semarang). Mémoire de maitrise en comptabilité, Semarang : Université Diponegoro.

Zhu, D.-S., Lin, L.H., Ni, F.-Y. (2002). A study of the relations among budgetary participation, the propensity to create budgetary slack, budgetary slack, and managerial performance. PanPacific Management Review 5 (1) : 77-91. 


\section{Annexe 1. Articles présentant un résultat empirique quantitatif pour le lien étudié}

\begin{tabular}{|c|c|c|c|c|}
\hline $\begin{array}{l}\text { Auteur(s), année } \\
\text { (support de } \\
\text { publication, } \\
\text { langue) }\end{array}$ & Échantillon & $\begin{array}{l}\text { Mesure de la } \\
\text { Participation }\end{array}$ & $\begin{array}{l}\text { Mesure de la } \\
\text { performance }\end{array}$ & $\begin{array}{c}\text { Codage de la } \\
\text { relation }\end{array}$ \\
\hline $\begin{array}{c}\text { Abdullah, } 1998 \\
\text { (Analisis, anglais) }\end{array}$ & 79 managers australiens & Milani (1975) & $\begin{array}{c}\text { Mahoney et al. } \\
(1963,1965)\end{array}$ & 1 \\
\hline $\begin{array}{l}\text { Adrianto, } 2008 \\
\text { (mémoire, } \\
\text { indonésien) }\end{array}$ & $\begin{array}{l}105 \text { managers } \\
\text { indonésiens }\end{array}$ & Échelle personnelle & Échelle personnelle & $\begin{array}{l}1 \\
1 \\
1 \\
1 \\
1 \\
1 \\
1 \\
1\end{array}$ \\
\hline $\begin{array}{c}\text { Affes et } \\
\text { Boujelbene, } 2010 \\
\text { (conf. AFC, } \\
\text { français) }\end{array}$ & 53 managers tunisiens & Milani (1975) & $\begin{array}{c}\text { Mahoney et al. } \\
(1963,1965)\end{array}$ & 1 \\
\hline $\begin{array}{c}\text { Agbejule et } \\
\text { Saarikoski, } 2006 \\
\text { (BAR, anglais) }\end{array}$ & 83 managers finlandais & Milani (1975) & $\begin{array}{c}\text { Mahoney et al. } \\
(1963,1965)\end{array}$ & $\begin{array}{c}-1 \\
1 \\
-1 \\
1 \\
1\end{array}$ \\
\hline $\begin{array}{c}\text { Alam et Mia, } 2006 \\
\text { (IJBR, anglais) }\end{array}$ & $\begin{array}{l}113 \text { managers d'une } \\
\text { organisation non } \\
\text { gouvernementale au } \\
\text { Bangladesh }\end{array}$ & Milani (1975) & $\begin{array}{c}\text { Mahoney et al. } \\
(1963,1965)\end{array}$ & 1 \\
\hline $\begin{array}{c}\text { Aranya, } 1990 \\
\text { (JMAR, anglais) }\end{array}$ & 97 managers canadiens & Échelle personnelle & Échelle personnelle & 1 \\
\hline $\begin{array}{c}\text { Arifin, } 2007 \\
\text { (KJBDM, } \\
\text { indonésien) }\end{array}$ & $\begin{array}{l}44 \text { managers } \\
\text { indonésiens }\end{array}$ & Échelle personnelle & Échelle personnelle & 1 \\
\hline $\begin{array}{l}\text { Bass et Leavitt, } \\
1963 \text { (MS, anglais) }\end{array}$ & $\begin{array}{c}3 \text { expérimentations avec } \\
36 \text { managers (supposés) } \\
\text { américains }\end{array}$ & Échelle personnelle & Échelle personnelle & $\begin{array}{l}1 \\
1 \\
1\end{array}$ \\
\hline $\begin{array}{l}\text { Bento et White, } \\
2006 \text { (AMA, } \\
\text { anglais) }\end{array}$ & 64 managers américains & $\begin{array}{l}\text { Chow et al. (1999), } \\
\text { adapté de Milani } \\
\text { (1975) }\end{array}$ & $\begin{array}{l}\text { Mahoney et al. } \\
(1963,1965)\end{array}$ & 1 \\
\hline $\begin{array}{l}\text { Breaux, } 2004 \\
\text { (thèse, anglais) }\end{array}$ & $\begin{array}{l}197 \text { membres de } \\
\text { l'American Institute of } \\
\text { Certified Public } \\
\text { Accountants }\end{array}$ & $\begin{array}{c}\text { Clinton et Hunton } \\
\text { (2001) }\end{array}$ & $\begin{array}{c}\text { Mahoney et al. } \\
(1963,1965)\end{array}$ & $\begin{array}{c}1 \\
-1\end{array}$ \\
\hline
\end{tabular}


Brownell, 1981

(TAR, anglais)

Brownell, $1982 a$

(JAR, anglais)

Brownell, $1982 b$ (TAR, anglais)

Brownell, 1983 (AOS, anglais)
Expérimentation : 46 Échelle personnelle Échelle personnelle étudiants, 48 managers américains

38 managers
américains
Hofstede (1967) et Milani (1975)

Mahoney et al. (1963, 1965)

40 managers américains

Hofstede (1967) et Milani (1975)

Mahoney et al. (1963, 1965)

46 managers (supposés) australiens
Hofstede (1967) et Milani (1975)

Mahoney et al. (1963, 1965)
1

1

Brownell, 1985

61 managers (supposés) australiens

Milani (1975)

Mahoney et al.

(1963, 1965)

Milani (1975)

Mahoney et al.

(1963, 1965)
Milani (1975)

Mahoney et al.

(1963, 1965)

Hofstede (1967) et

Milani (1975)

Mahoney et al.

(1963, 1965)

Version modifiée Échelle personnelle de Swieringa et

Moncur (1975)

McInnes, 1986 (supposés) américains

(TAR, anglais)

Brownell et

Merchant, 1990

(JAR, anglais)

146 gestionnaires de production (supposés) américains

Byrne et Damon,

Expérimentation : 83

Échelle personnelle Échelle personnelle anglais)

Chalos et Haka, Expérimentation : 240 Échelle personnelle Échelle personnelle

$$
\text { américains en Master of }
$$

\section{Business}

Administration

Chalos et Poon 72 managers (supposés)

2001 (AMA, américains

Milani (1975) anglais)
Mahoney et al.

(1963, 1965) 
Chenhall et Brownell, 1988 (AOS, anglais)

Cherrington et Cherrington, 1973 (ERASS, anglais)

Chong et Bateman, 79 managers australiens 2000 (AABR, anglais)

Chong et Chong, 79 managers australiens $2002 a$ (BRA, anglais)

Chong et Chong, 79 managers australiens $2002 b$ (PAR, anglais)

Chong, Eggleton et 77 managers australiens Leong, 2005 (BAR, anglais)

Chong, Eggleton et 74 managers australiens Adaptée de Milani Leong, 2006 (AA, anglais)

$\begin{array}{cc}\text { Damanik, } 2009 & 95 \text { managers } \\ \text { (mémoire, } & \text { indonésiens } \\ \text { indonésien) } & \end{array}$

Damayanti, 2007
(JAAI, indonésien)

$\begin{array}{lc}\text { Din, } 2008 & 175 \text { managers } \\ \text { (mémoire, } & \text { indonésiens }\end{array}$
indonésien)

Dunk, 1989 (AOS, 26 managers du nord du anglais)

Royaume-Uni

Dunk, 1990 (AOS, 26 managers du nord du anglais)

Royaume-Uni

Dunk, 1993 (TAR, 78 managers australiens anglais)

Dunk,1995a (SJM, 78 managers australiens anglais)

Eker, 2009 (Ä̈SD, 150 managers turcs anglais)

Etemadi, Dilami, 256 managers iraniens Bazaz et
Milani (1975) Échelle personnelle

Milani (1975)

Mahoney et al.

(1963, 1965)

$-1$

1

$-1$

$-1$

Milani (1975)

Mahoney et al. (1963, 1965)

Milani (1975)

Kenis (1979)

Milani (1975)

Échelle personnelle, cohérente avec celle de Merchant (1981)

$-1$

$-1$

$-1$

Échelle personnelle, cohérente avec celle de Merchant (1981)

Milani (1975)

Mahoney et al. (1963, 1965)

Milani (1975)

Mahoney et al. (1963, 1965)

Milani (1975)

Mahoney et al. (1963, 1965)

Milani (1975)

Mahoney et al. (1963, 1965)

Milani (1975)

Mahoney et al. (1963, 1965)

Milani (1975)

Milani (1975)

Mahoney et al. (1963, 1965)

Adaptées de

Merchant (1981,

1984) et Brownell et Merchant (1990)

Milani (1975)

Mahoney et al. (1963, 1965)

Milani (1975)

Mahoney et al. (1963, 1965)
1 


\section{Parameswaran, \\ 2009 (AIA, anglais)}

Frucot et Shearon, 83 managers mexicains 1991 (TAR, anglais)

Frucot et White, 2006 (AMA, anglais)

Godener et 155 managers français Fornerino, 2009 (conf. AFC, français)

Govindarajan, 77 managers (supposés) 1986 (DS, anglais)

Gul, Tsui, Fong et 37 managers de Hong Kwok, 1995 (ABR, anglais)

Hafiz, 2007 (mémoire, indonésien)

Hassel et 36 managers finlandais Cunningham, 1996 (MIR, anglais) 178 managers américains

Milani (1975)

Milani (1975)

Adaptée de

Godener et

Fornerino (2005)

Swieringa et Moncur (1975)

Milani (1975) Kong

32 managers indonésiens

Milani (1975)

Adaptée de Milani

(1975)

$$
\text { étrangers }
$$

Mahoney et al. $(1963,1965)$

Mahoney et al. $(1963,1965)$ et Heneman (1974)

Adaptée de Govindarajan et Gupta (1985)

Mahoney et al. (1963, 1965)

Mahoney et al. (1963, 1965)

Mahoney et al. (1963, 1965)

Adaptée de $-1$ Govindarajan (1984) $\quad-1$ et Gupta et 1 Govindarajan (1984) 1

$-1$

Heath et Brown, 256 managers

Milani (1975) 2007 (JABR, américains anglais)

Hirst, 1987 (AJM, 44 managers australiens anglais)

Hoque et Brosnan, 55 managers australiens

1

(1)

(1)

(1)




\begin{tabular}{|c|c|c|c|c|}
\hline 2007 (WP, anglais) & & & $(1963,1965)$ & 1 \\
\hline $\begin{array}{l}\text { Iriyadi et Gurd, } \\
1998 \text { (ARA, } \\
\text { anglais) }\end{array}$ & $\begin{array}{l}48 \text { managers } \\
\text { indonésiens }\end{array}$ & Milani (1975) & $\begin{array}{l}\text { Mahoney et al. } \\
(1963,1965)\end{array}$ & $\begin{array}{l}1 \\
1\end{array}$ \\
\hline $\begin{array}{l}\text { Jermias et } \\
\text { Setiawan, } 2008 \\
\text { (IJA, anglais) }\end{array}$ & $\begin{array}{l}204 \text { managers d'une } \\
\text { administration } \\
\text { indonésienne }\end{array}$ & $\begin{array}{l}\text { Adaptée de Vroom } \\
\text { et Mann (1960) }\end{array}$ & $\begin{array}{c}\text { Adaptée de } \\
\text { Mahoney et al. } \\
(1963,1965)\end{array}$ & $\begin{array}{l}-1 \\
-1\end{array}$ \\
\hline $\begin{array}{c}\text { Kenis, } 1979 \text { (TAR, } \\
\text { anglais) }\end{array}$ & $\begin{array}{l}169 \text { managers } \\
\text { américains }\end{array}$ & Échelle personnelle & Échelle personnelle & 1 \\
\hline $\begin{array}{c}\text { Khalil, } 2009 \\
\text { (mémoire, anglais) }\end{array}$ & $\begin{array}{l}195 \text { managers } \\
\text { malaisiens }\end{array}$ & Milani (1975) & $\begin{array}{c}\text { Mahoney et al. } \\
(1963,1965)\end{array}$ & 1 \\
\hline $\begin{array}{l}\text { Kobori, } 2006 \\
\text { (mémoire, } \\
\text { japonais) }\end{array}$ & 81 managers taïwanais & Hofstede (1967) & $\begin{array}{l}\text { Mahoney et al. } \\
(1963,1965)\end{array}$ & $\begin{array}{l}1 \\
1\end{array}$ \\
\hline $\begin{array}{c}\text { Kren, } 1992 \\
\text { (TAR, anglais) }\end{array}$ & 80 managers américains & Milani (1975) & $\begin{array}{l}\text { Mahoney et al. } \\
(1963,1965)\end{array}$ & $\begin{array}{c}1 \\
1 \\
-1\end{array}$ \\
\hline $\begin{array}{l}\text { Latif, } 2007 \\
\text { (mémoire, } \\
\text { indonésien) }\end{array}$ & $\begin{array}{l}55 \text { managers } \\
\text { indonésiens }\end{array}$ & Milani (1975) & $\begin{array}{l}\text { Mahoney et al. } \\
(1963,1965)\end{array}$ & 1 \\
\hline $\begin{array}{c}\text { Lau et Buckland, } \\
2000 \text { (ABR, } \\
\text { anglais) }\end{array}$ & 71 managers norvégiens & Milani (1975) & $\begin{array}{l}\text { Mahoney et al. } \\
(1963,1965)\end{array}$ & -1 \\
\hline $\begin{array}{c}\text { Lau et Lim, 2002a } \\
\text { (BAR, anglais) }\end{array}$ & 83 managers australiens & Milani (1975) & $\begin{array}{l}\text { Mahoney et al. } \\
(1963,1965)\end{array}$ & 1 \\
\hline $\begin{array}{l}\text { Lau et Lim, 2002b } \\
\quad \text { (AIA, anglais) }\end{array}$ & 83 managers australiens & Milani (1975) & $\begin{array}{l}\text { Mahoney et al. } \\
(1963,1965)\end{array}$ & $\begin{array}{c}-1 \\
1 \\
1 \\
1 \\
1\end{array}$ \\
\hline $\begin{array}{l}\text { Lau, Low et } \\
\text { Eggleton, } 1995 \\
\text { (AOS, anglais) }\end{array}$ & $\begin{array}{l}112 \text { managers } \\
\text { singapouriens }\end{array}$ & Milani (1975) & $\begin{array}{l}\text { Mahoney et al. } \\
(1963,1965)\end{array}$ & $\begin{array}{c}-1 \\
-1 \\
-1 \\
1 \\
1 \\
1 \\
-1\end{array}$ \\
\hline $\begin{array}{c}\text { Lau, Low et } \\
\text { Eggleton, } 1997 \\
\text { (AAAJ, anglais) }\end{array}$ & $\begin{array}{c}90 \text { managers australiens } \\
\text { et } 107 \text { managers } \\
\text { singapouriens }\end{array}$ & Milani (1975) & $\begin{array}{l}\text { Mahoney et al. } \\
(1963,1965)\end{array}$ & $\begin{array}{c}-1 \\
-1 \\
-1 \\
1 \\
-1 \\
1 \\
-1 \\
-1\end{array}$ \\
\hline
\end{tabular}




\begin{tabular}{|c|c|c|c|c|}
\hline & & & & $\begin{array}{l}-1 \\
-1\end{array}$ \\
\hline $\begin{array}{l}\text { Lau et Tan, } 1998 \\
\text { (MAR, anglais) }\end{array}$ & $\begin{array}{c}104 \text { managers } \\
\text { australiens et } 85 \\
\text { managers singapouriens }\end{array}$ & Milani (1975) & $\begin{array}{l}\text { Mahoney et al. } \\
(1963,1965)\end{array}$ & $\begin{array}{c}1 \\
1 \\
-1\end{array}$ \\
\hline $\begin{array}{c}\text { Leach-López, } \\
\text { Stammerjohan et } \\
\text { Lee, } 2009 \text { (MRN, } \\
\text { anglais) }\end{array}$ & $\begin{array}{l}71 \text { managers sud- } \\
\text { coréens }\end{array}$ & Milani (1975) & $\begin{array}{l}\text { Mahoney et al. } \\
(1963,1965)\end{array}$ & $\begin{array}{l}1 \\
1 \\
1\end{array}$ \\
\hline $\begin{array}{l}\text { Leach-López, } \\
\text { Stammerjohan et } \\
\text { McNair, 2007 } \\
\text { (JMAR, anglais) }\end{array}$ & $\begin{array}{c}98 \text { managers américains } \\
\text { et } 45 \text { managers } \\
\text { mexicains }\end{array}$ & Milani (1975) & $\begin{array}{l}\text { Mahoney et al. } \\
(1963,1965)\end{array}$ & $\begin{array}{l}1 \\
1\end{array}$ \\
\hline $\begin{array}{c}\text { Leach-López, } \\
\text { Stammerjohan et } \\
\text { Rigsby, } 2008 \text { (conf. } \\
\text { EABR, anglais) }\end{array}$ & $\begin{array}{c}45 \text { managers américains } \\
\text { et } 89 \text { managers } \\
\text { mexicains }\end{array}$ & Milani (1975) & $\begin{array}{l}\text { Mahoney et al. } \\
(1963,1965)\end{array}$ & $\begin{array}{l}1 \\
1\end{array}$ \\
\hline $\begin{array}{l}\text { Libby, } 1999 \text { (AOS, } \\
\text { anglais) }\end{array}$ & $\begin{array}{l}\text { Expérimentation : } 171 \\
\text { étudiants canadiens }\end{array}$ & Échelle personnelle & Échelle personnelle & $\begin{array}{c}1 \\
-1 \\
1\end{array}$ \\
\hline $\begin{array}{l}\text { Lindquist, } 1995 \\
\text { (JMAR, anglais) }\end{array}$ & $\begin{array}{c}\text { Expérimentation : } 86 \\
\text { étudiants (supposés) } \\
\text { américains }\end{array}$ & Échelle personnelle & Échelle personnelle & $\begin{array}{c}-1 \\
1 \\
-1 \\
-1 \\
1 \\
-1 \\
1 \\
1 \\
-1 \\
1 \\
-1 \\
1\end{array}$ \\
\hline $\begin{array}{l}\text { Merchant, } 1981 \\
\text { (TAR, anglais) }\end{array}$ & $\begin{array}{l}19 \text { entreprises } \\
\text { électroniques } \\
\text { (supposées) } \\
\text { américaines }\end{array}$ & Échelle personnelle & Échelle personnelle & $\begin{array}{c}1 \\
-1 \\
1 \\
1 \\
-1 \\
-1 \\
1 \\
1 \\
1\end{array}$ \\
\hline $\begin{array}{c}\text { Merchant, } 1984 \\
\text { (AOS, anglais) }\end{array}$ & $\begin{array}{c}170 \text { managers } \\
\text { (supposés) américains }\end{array}$ & Échelle personnelle & Échelle personnelle & $\begin{array}{l}1 \\
1 \\
1 \\
1 \\
1 \\
1\end{array}$ \\
\hline
\end{tabular}




\begin{tabular}{|c|c|c|c|c|}
\hline & & & & $\begin{array}{c}-1 \\
1 \\
-1\end{array}$ \\
\hline $\begin{array}{c}\text { Mia, } 1988(A O S, \\
\text { anglais) }\end{array}$ & 51 managers australiens & Milani (1975) & Échelle personnelle & $\begin{array}{l}-1 \\
-1\end{array}$ \\
\hline $\begin{array}{c}\text { Mia, } 1989(A O S, \\
\text { anglais) }\end{array}$ & $\begin{array}{l}62 \text { managers néo- } \\
\text { zélandais }\end{array}$ & Milani (1975) & Échelle personnelle & 1 \\
\hline $\begin{array}{c}\text { Mia et Patiar, } 2002 \\
\text { (JHTR, anglais) }\end{array}$ & 52 managers australiens & Milani (1975) & $\begin{array}{l}\text { Mahoney et al. } \\
(1963,1965)\end{array}$ & $\begin{array}{l}-1 \\
-1 \\
-1 \\
-1 \\
-1 \\
-1\end{array}$ \\
\hline $\begin{array}{c}\text { Milani, } 1975 \text { (TAR, } \\
\text { anglais) }\end{array}$ & $\begin{array}{c}82 \text { contremaîtres } \\
\text { (supposés) américains }\end{array}$ & Échelle personnelle & Échelle personnelle & $\begin{array}{l}1 \\
1 \\
1 \\
1 \\
1 \\
1 \\
1 \\
1 \\
1 \\
1 \\
1 \\
1\end{array}$ \\
\hline $\begin{array}{l}\text { Ni et Su, } 2001 \\
\text { (CMQ, chinois) }\end{array}$ & 205 managers taïwanais & Milani (1975) & $\begin{array}{l}\text { Mahoney et al. } \\
(1963,1965)\end{array}$ & $\begin{array}{c}1 \\
-1 \\
1\end{array}$ \\
\hline $\begin{array}{l}\mathrm{Ni}, \mathrm{Su}, \text { Chung et } \\
\text { Cheng, } 2005 \text { (conf. } \\
\text { A-P, anglais) }\end{array}$ & 155 managers taïwanais & Milani (1975) & $\begin{array}{l}\text { Mahoney et al. } \\
(1963,1965)\end{array}$ & $\begin{array}{l}1 \\
1 \\
1 \\
1\end{array}$ \\
\hline $\begin{array}{l}\text { Nor, } 2007 \text { (conf. } \\
\text { SNA, indonésien) }\end{array}$ & $\begin{array}{l}101 \text { managers } \\
\text { indonésiens }\end{array}$ & Milani (1975) & $\begin{array}{l}\text { Mahoney et al. } \\
(1963,1965)\end{array}$ & 1 \\
\hline $\begin{array}{l}\text { Nouri, Blau et } \\
\text { Shahid, } 1995 \\
\text { (AMA, anglais) }\end{array}$ & $\begin{array}{l}135 \text { managers } \\
\text { américains }\end{array}$ & Milani (1975) & $\begin{array}{c}\text { Mahoney et al. } \\
(1963,1965) \\
\text { Gupta et } \\
\text { Govindarajan (1984) }\end{array}$ & $\begin{array}{l}-1 \\
-1\end{array}$ \\
\hline $\begin{array}{c}\text { Nouri et Parker, } \\
1998 \text { (AOS, } \\
\text { anglais) }\end{array}$ & $\begin{array}{l}135 \text { managers } \\
\text { américains }\end{array}$ & Milani (1975) & $\begin{array}{l}\text { Govindarajan et } \\
\text { Gupta (1985) }\end{array}$ & 1 \\
\hline $\begin{array}{c}\text { Orpen, } 1992 \text { (JSP, } \\
\text { anglais) }\end{array}$ & $\begin{array}{l}136 \text { managers } \\
\text { australiens }\end{array}$ & Milani (1975) & Échelle personnelle & $\begin{array}{l}1 \\
1\end{array}$ \\
\hline $\begin{array}{l}\text { Otley et Pollanen, } \\
2000(A O S,\end{array}$ & $\begin{array}{l}121 \text { managers } \\
\text { d'administration }\end{array}$ & Milani (1975) & $\begin{array}{l}\text { Mahoney et al. } \\
(1963,1965)\end{array}$ & $\begin{array}{l}1 \\
1\end{array}$ \\
\hline
\end{tabular}




\begin{tabular}{|c|c|c|c|c|}
\hline anglais) & $\begin{array}{l}\text { d'universités } \\
\text { canadiennes }\end{array}$ & & & -1 \\
\hline $\begin{array}{l}\text { Parker et Kyj, } \\
2006 \text { (AOS, } \\
\text { anglais) }\end{array}$ & $\begin{array}{c}70 \text { managers (supposés) } \\
\text { américains }\end{array}$ & Milani (1975) & $\begin{array}{l}\text { Mahoney et al. } \\
(1963,1965)\end{array}$ & 1 \\
\hline $\begin{array}{l}\text { Puspaningsih, } \\
2003 \text { (JAAI, } \\
\text { indonésien) }\end{array}$ & $\begin{array}{l}58 \text { managers } \\
\text { indonésiens }\end{array}$ & $\begin{array}{c}\text { Adaptée de Milani } \\
\text { (1975) }\end{array}$ & $\begin{array}{l}\text { Mahoney et al. } \\
(1963,1965)\end{array}$ & 1 \\
\hline $\begin{array}{l}\text { Puspaningsih, } \\
2004 \text { (JAAI, } \\
\text { indonésien) }\end{array}$ & $\begin{array}{l}58 \text { managers } \\
\text { indonésiens }\end{array}$ & $\begin{array}{c}\text { Adaptée de Milani } \\
\text { (1975) }\end{array}$ & $\begin{array}{l}\text { Mahoney et al. } \\
(1963,1965)\end{array}$ & 1 \\
\hline $\begin{array}{l}\text { Qi, } 2010 \text { (thèse, } \\
\text { anglais) }\end{array}$ & 75 managers chinois & $\begin{array}{c}\text { Adaptée de Milani } \\
\text { (1975) }\end{array}$ & $\begin{array}{l}\text { Mahoney et al. } \\
(1963,1965)\end{array}$ & $\begin{array}{l}1 \\
1\end{array}$ \\
\hline $\begin{array}{l}\text { Quirin, O’Bryan et } \\
\text { Donnelly, } 2000 \\
\text { (AMA, anglais) }\end{array}$ & $\begin{array}{l}107 \text { managers } \\
\text { américains }\end{array}$ & Milani (1975) & $\begin{array}{l}\text { Mahoney et al. } \\
(1963,1965)\end{array}$ & 1 \\
\hline $\begin{array}{l}\text { Quirin, O’Bryan et } \\
\text { Donnelly, } 2004 \\
\text { (AMA, anglais) }\end{array}$ & 98 managers américains & Milani (1975) & $\begin{array}{l}\text { Mahoney et al. } \\
(1963,1965)\end{array}$ & 1 \\
\hline $\begin{array}{c}\text { Sardjito et } \\
\text { Muthaher, 2007 } \\
\text { (conf. SNA, } \\
\text { indonésien) }\end{array}$ & $\begin{array}{l}102 \text { managers publics } \\
\text { indonésiens }\end{array}$ & Milani (1975) & $\begin{array}{l}\text { Mahoney et al. } \\
(1963,1965)\end{array}$ & $\begin{array}{l}1 \\
-1 \\
-1\end{array}$ \\
\hline $\begin{array}{l}\text { Shields, Deng et } \\
\text { Kato, } 2000 \text { (AOS, } \\
\text { anglais) }\end{array}$ & 358 ingénieurs japonais & $\begin{array}{l}\text { Adaptée de Shields } \\
\text { et Young (1993) }\end{array}$ & Échelle personnelle & 1 \\
\hline $\begin{array}{c}\text { Shields et Young } \\
1993 \text { (JMAR, } \\
\text { anglais) }\end{array}$ & $\begin{array}{l}98 \text { contrôleurs de } \\
\text { gestion américains }\end{array}$ & Échelle personnelle & Échelle personnelle & 1 \\
\hline $\begin{array}{l}\text { tevanny et Merita, } \\
2005 \text { (mémoire, } \\
\text { indonésien) }\end{array}$ & $\begin{array}{l}16 \text { managers } \\
\text { indonésiens }\end{array}$ & Échelle personnelle & Échelle personnelle & 1 \\
\hline $\begin{array}{l}\text { Su, } 2001 \text { (résultats } \\
\text { principaux } \\
\text { communiqués par } \\
\text { Ni, Su et Su, 2003) } \\
\text { (mémoire et conf. } \\
\text { ICCM, chinois et } \\
\text { anglais) }\end{array}$ & 194 managers taïwanais & Hofstede (1967) & $\begin{array}{l}\text { Mahoney et al. } \\
(1963,1965)\end{array}$ & $\begin{array}{c}-1 \\
-1 \\
-1 \\
1 \\
-1 \\
-1 \\
-1\end{array}$ \\
\hline $\begin{array}{l}\text { Su, } 2008 \text { (thèse, } \\
\text { chinois) }\end{array}$ & 164 managers taïwanais & Milani (1975) & $\begin{array}{l}\text { Mahoney et al. } \\
(1963,1965)\end{array}$ & 1 \\
\hline $\begin{array}{l}\text { Su et Lin, } 2007 \\
\text { (WP, anglais) }\end{array}$ & 168 managers taïwanais & Milani (1975) & $\begin{array}{l}\text { Mahoney et al. } \\
(1963,1965)\end{array}$ & 1 \\
\hline Subramaniam et & 114 managers & Milani (1975) & Mahoney et al. & -1 \\
\hline
\end{tabular}




\begin{tabular}{|c|c|c|c|c|}
\hline $\begin{array}{c}\text { Ashkanasy, } 2001 \\
\text { (AJM, anglais) }\end{array}$ & australiens & & $(1963,1965)$ & \\
\hline $\begin{array}{l}\text { Sumarno, } 2006 \\
\text { (mémoire, } \\
\text { indonésien) }\end{array}$ & $\begin{array}{l}100 \text { managers } \\
\text { indonésiens }\end{array}$ & $\begin{array}{l}\text { Adaptée de Shields } \\
\text { et Young (1993) }\end{array}$ & $\begin{array}{l}\text { Adaptée de Shields, } \\
\text { Deng et Kato (2000) }\end{array}$ & 1 \\
\hline $\begin{array}{l}\text { Syah, } 2009 \\
\text { (mémoire, } \\
\text { indonésien) }\end{array}$ & $\begin{array}{l}33 \text { managers } \\
\text { indonésiens }\end{array}$ & $\begin{array}{l}\text { Échelle personnelle } \\
\text { (supposée) }\end{array}$ & $\begin{array}{l}\text { Échelle personnelle } \\
\text { (supposée) }\end{array}$ & -1 \\
\hline $\begin{array}{l}\text { Taylor, Abdul- } \\
\text { Hamid et Mohd- } \\
\text { Sanusi, } 2008 \\
\text { (JAMAR, anglais) }\end{array}$ & $\begin{array}{c}81 \text { managers } \\
\text { d'administrations } \\
\text { territoriales } \\
\text { malaisiennes }\end{array}$ & Milani (1975) & $\begin{array}{c}\text { Adaptée de } \\
\text { Mahoney et al. } \\
(1963,1965)\end{array}$ & $\begin{array}{l}1 \\
1\end{array}$ \\
\hline $\begin{array}{c}\text { Tiller, } 1983 \text { (JAR, } \\
\text { anglais) }\end{array}$ & $\begin{array}{c}\text { Expérimentation : } 150 \\
\text { étudiants (supposés) } \\
\text { américains }\end{array}$ & Échelle personnelle & Échelle personnelle & 1 \\
\hline $\begin{array}{l}\text { Tintri, } 2002 \text { (JEB, } \\
\text { indonésien) }\end{array}$ & $\begin{array}{c}53 \text { managers (supposés) } \\
\text { indonésiens }\end{array}$ & Milani (1975) & $\begin{array}{l}\text { Mahoney et al. } \\
(1963,1965)\end{array}$ & -1 \\
\hline $\begin{array}{c}\text { Tsamenyi et Mills, } \\
2002 \text { (JTMD, } \\
\text { anglais) }\end{array}$ & 89 managers ghanéens & Milani (1975) & $\begin{array}{l}\text { Mahoney et al. } \\
(1963,1965)\end{array}$ & $\begin{array}{l}1 \\
1\end{array}$ \\
\hline $\begin{array}{l}\text { Tsui, } 2001 \text { (IJA, } \\
\text { anglais) }\end{array}$ & $\begin{array}{l}89 \text { managers de Hong } \\
\text { Kong }\end{array}$ & Milani (1975) & $\begin{array}{l}\text { Mahoney et al. } \\
(1963,1965)\end{array}$ & $\begin{array}{l}1 \\
1 \\
1 \\
1 \\
1 \\
1\end{array}$ \\
\hline $\begin{array}{l}\text { Wentzel, } 2002 \\
\text { (BRA, anglais) }\end{array}$ & $\begin{array}{c}74 \text { managers (supposés) } \\
\text { américains }\end{array}$ & Milani (1975) & $\begin{array}{l}\text { Mahoney et al. } \\
(1963,1965)\end{array}$ & 1 \\
\hline $\begin{array}{l}\text { Winata et Mia, } \\
2005 \text { (IJHM, } \\
\text { anglais) }\end{array}$ & 74 managers australiens & $\begin{array}{c}\text { Adaptée de Milani } \\
\text { (1975) }\end{array}$ & Échelle personnelle & 1 \\
\hline $\begin{array}{l}\text { Wu, } 2004 \text { (publié } \\
\text { oar Chang, Chang, } \\
\text { Chang et Wu, } \\
\text { 2007) (IJAS, } \\
\text { chinois) }\end{array}$ & 104 managers taïwanais & Kenis (1979) & $\begin{array}{l}\text { Govindarajan et } \\
\text { Gupta (1985) }\end{array}$ & $\begin{array}{l}1 \\
1 \\
1 \\
1\end{array}$ \\
\hline $\begin{array}{l}\text { Yahya, Ahmad et } \\
\text { Fatima, } 2008 \\
\text { (IJPSM, anglais) }\end{array}$ & $\begin{array}{l}111 \text { managers du } \\
\text { ministère de la défense } \\
\text { malaisien }\end{array}$ & Milani (1975) & $\begin{array}{l}\text { Mahoney et al. } \\
(1963,1965)\end{array}$ & 1 \\
\hline $\begin{array}{c}\text { Yuen, } 2007 \text { (MAJ, } \\
\text { anglais) }\end{array}$ & $\begin{array}{l}216 \text { managers d'un } \\
\text { service public chinois }\end{array}$ & $\begin{array}{l}\text { Échelle (supposée) } \\
\text { de Milani (1975) }\end{array}$ & $\begin{array}{l}\text { Mahoney et al. } \\
(1963,1965)\end{array}$ & 1 \\
\hline $\begin{array}{c}\text { Yuen et Cheung, } \\
2003 \text { (JAMAR, } \\
\text { anglais) }\end{array}$ & 165 managers chinois & Milani (1975) & $\begin{array}{l}\text { Mahoney et al. } \\
(1963,1965)\end{array}$ & -1 \\
\hline
\end{tabular}


Yunita, 2009

(mémoire,

indonésien)

$\mathrm{Zhu}$, Lin et $\mathrm{Ni}$

2002 (PMR,

anglais)
75 officiels d'une Wentzel (2002) Heneman (1974)

université indonésienne
1

Mahoney et al.

1

Note : Les supports de publication sont résumés par leurs acronymes. «WP » est l'acronyme de working paper pour une étude non publiée et « conf. » rend compte du fait qu'il s'agit d'un article communiqué et non publié.

\section{Annexe 2. Détermination du score de qualité}

\begin{tabular}{|c|c|c|c|c|c|c|}
\hline $\begin{array}{c}\text { Auteurs, date } \\
\text { (support de } \\
\text { publication, langue) }\end{array}$ & $\begin{array}{l}\text { Sélection } \\
\text { aléatoire }\end{array}$ & $\begin{array}{c}\text { Score de } \\
\text { « qualité » }\end{array}$ & $\begin{array}{c}\text { Taille } \\
\text { d'échantillon }\end{array}$ & $\begin{array}{l}\text { Pertinence } \\
\text { des } \\
\text { échelles }\end{array}$ & $\begin{array}{l}\text { Précision } \\
\text { du design }\end{array}$ & $\begin{array}{l}\text { Validité } \\
\text { externe }\end{array}$ \\
\hline $\begin{array}{c}\text { Adrianto, } 2008 \\
\text { (mémoire, indonésien) }\end{array}$ & & 3,29 & 1,29 & 0,00 & 1,00 & 1,00 \\
\hline $\begin{array}{l}\text { Agbejule et Saarikoski, } \\
2006 \text { (BAR, anglais) }\end{array}$ & & 4,22 & 1,02 & 1,00 & 1,20 & 1,00 \\
\hline $\begin{array}{l}\text { Alam et Mia, 2006 } \\
\text { (IJBR, anglais) }\end{array}$ & & 4,39 & 1,39 & 1,00 & 1,00 & 1,00 \\
\hline $\begin{array}{c}\text { Aranya, } 1990 \text { (JMAR, } \\
\text { anglais) }\end{array}$ & & 3,40 & 1,20 & 0,00 & 1,20 & 1,00 \\
\hline $\begin{array}{c}\text { Arifin, } 2007 \text { (KJBDM, } \\
\text { indonésien) }\end{array}$ & & 2,34 & 0,54 & 0,00 & 0,80 & 1,00 \\
\hline $\begin{array}{c}\text { Breaux, } 2004 \text { (thèse, } \\
\text { anglais) }\end{array}$ & $*$ & 5,03 & 2,43 & 0,50 & 1,10 & 1,00 \\
\hline $\begin{array}{c}\text { Brownell, } 1982 \text { a (JAR, } \\
\text { anglais) }\end{array}$ & & 3,22 & 0,47 & 0,75 & 1,00 & 1,00 \\
\hline $\begin{array}{c}\text { Brownell, } 1982 b \text { (TAR, } \\
\text { anglais) }\end{array}$ & & 3,44 & 0,49 & 0,75 & 1,20 & 1,00 \\
\hline $\begin{array}{c}\text { Brownell, } 1985 \text { (JAR, } \\
\text { anglais) }\end{array}$ & & 3,75 & 0,75 & 1,00 & 1,00 & 1,00 \\
\hline $\begin{array}{l}\text { Brownell et Dunk, } \\
1991 \text { (AOS, anglais) }\end{array}$ & $*$ & 3,84 & 0,54 & $1,00^{\#}$ & 1,30 & 1,00 \\
\hline $\begin{array}{l}\text { Brownell et Dunk, } \\
1991 \text { (AOS, anglais) }\end{array}$ & $*$ & 3,73 & 0,43 & $1,00^{\#}$ & 1,30 & 1,00 \\
\hline $\begin{array}{c}\text { Brownell et Hirst, } 1986 \\
\text { (JAR, anglais) }\end{array}$ & & 3,94 & 0,94 & 1,00 & 1,00 & 1,00 \\
\hline $\begin{array}{c}\text { Brownell et McInnes, } \\
1986 \text { (TAR, anglais) }\end{array}$ & & 4,33 & 1,33 & 1,00 & 1,00 & 1,00 \\
\hline $\begin{array}{c}\text { Brownell et Merchant, } \\
1990 \text { (JAR, anglais) }\end{array}$ & & 3,70 & 1,80 & 0,00 & 0,90 & 1,00 \\
\hline $\begin{array}{l}\text { Chalos et Poon, } 2001 \\
\text { (AMA, anglais) }\end{array}$ & $*$ & 3,79 & 0,89 & $1,00^{\#}$ & 0,90 & 1,00 \\
\hline $\begin{array}{c}\text { Chenhall et Brownell, } \\
1988 \text { (AOS, anglais) }\end{array}$ & & 2,91 & 0,41 & 0,50 & 1,00 & 1,00 \\
\hline $\begin{array}{c}\text { Chong et Bateman, } \\
2000 \text { (AABR, anglais) }\end{array}$ & $*$ & 3,97 & 0,97 & $1,00^{\#}$ & 1,00 & 1,00 \\
\hline
\end{tabular}


Chong et Chong,

$2002 b$ (PAR, anglais)

Chong, Eggleton et

Leong, 2005 (BAR, anglais)

Chong, Eggleton et

Leong, 2006 (AA, anglais)

Damanik, 2009

(mémoire, indonésien)

Damayanti, 2007

(JAAI, indonésien)

Din, 2008 (mémoire, indonésien)

Dunk, 1990 (AOS, anglais)

Dunk, 1993 (TAR, anglais)

Dunk, 1995 a (SJM, anglais)

Dunk, 1995 a (SJM, anglais)

Eker, 2009 (AÜSD, anglais)

Etemadi, Dilami, Bazaz et

Parameswaran, 2009 (AIA, anglais)

Frucot et Shearon, 1991 (TAR, anglais)

Frucot et White, 2006 (AMA, anglais)

Govindarajan, 1986 (DS, anglais)

Gul, Tsui, Fong et Kwok, 1995 (ABR, anglais)

Hafiz, 2007 (mémoire, indonésien)

Hassel et Cunningham, 1996 (MIR, anglais)

Hassel et Cunningham, 1996 (MIR, anglais)

Heath et Brown, 2007 (JABR, anglais)

Hirst, 1987 (AJM, anglais)

Hoque et Brosnan, 2007 (WP, anglais)

Iriyadi et Gurd, 1998
3,77

0,97

0,50

1,30

1,00

3,75

0,95

0,50

1,30

1,00

3,26

0,91

0,25

1,10

1,00

4,17

1,17

$1,00^{\#}$

1,00

1,00

4,51

1,61

1,00

0,90

1,00

5,26

2,16

1,00

1,10

1,00

3,42

0,32

$1,00^{\#}$

1,10

1,00

4,26

0,96

$1,00^{\#}$

1,30

1,00

3,14

0,54

0,50

1,10

1,00

3,02

0,42

0,50

1,10

1,00

4,85

1,85

1,00

1,00

1,00

6,25

3,15

1,00

1,10

1,00

4,02

1,02

1,00

1,00

1,00

4,99

2,19

1,00

0,80

1,00

3,65

0,95

0,50

1,20

1,00

3,26

0,46

1,00

0,80

1,00

3,29

0,39

1,00

0,90

1,00

3,09

0,44

0,25

1,40

1,00

3,03

0,38

0,25

1,40

1,00

5,85

3,15

0,50

1,20

1,00

3,54

0,54

1,00

1,00

1,00

3,78

0,68

$1,00^{\#}$

1,10

1,00

3,69

0,59

$1,00^{\#}$

1,10

1,00 
(ARA, anglais)

Jermias et Setiawan,

2008 (IJA, anglais)

5,06

2,51

0,25

1,30

1,00

Kenis, 1979 (TAR, anglais)

4,18

2,08

0,00

1,10

1,00

Kobori, 2006

(mémoire, japonais)

3,60

1,00

0,50

1,10

1,00

Kren, 1992 (TAR,

4,09

0,99

$1,00^{\#}$

1,10

1,00

3,68

0,68

1,00

1,00

1,00

Lau et Buckland, 2000

4,08

0,88

$1,00^{\#}$

1,20

1,00

Lau et Lim, 2002b

3,61

0,51

$1,00^{\#}$

1,10

1,00

Lau et Lim, 2002b (AIA, anglais)

3,62

0,52

$1,00^{\#}$

1,10

1,00

Lau, Low et Eggleton,

1995 (AOS, anglais)

4,04

0,74

$1,00^{\#}$

1,30

1,00

Lau, Low et Eggleton,

3,94

0,64

$1,00^{\#}$

1,30

1,00

Lau, Low et Eggleton,

4,41

1,11

$1,00^{\#}$

1,30

1,00

Lau, Low et Eggleton,

4,62

1,32

$1,00^{\#}$

1,30

1,00

Lau et Tan, 1998

(MAR, anglais)

5,43

2,33

$1,00^{\#}$

1,10

1,00

Leach-López,

Stammerjohan et Lee,

3,67

0,67

1,00

1,00

1,00

2009 (MRN, anglais)

Leach-López,

Stammerjohan et Lee,

3,21

0,21

1,00

1,00

1,00

2009 (MRN, anglais)

Leach-López,

Stammerjohan et

McNair, 2007 (JMAR,

1,21

1,00

1,00

1,00

anglais)

Leach-López,

Stammerjohan et

McNair, 2007 (JMAR,

3,55

0,55

1,00

1,00

1,00

anglais)

Mia, 1988 (AOS,

$$
\text { anglais) }
$$

3,13

0,63

0,50

1,00

1,00

Mia, 1989 (AOS, anglais)

3,26

0,76

0,50

1,00

1,00

Mia et Patiar, 2002

(JHTR, anglais)

3,84

0,64

1,00

1,20

1,00

Ni et Su, 2001 (CMQ, chinois)

4,11

1,31

$1,00^{\#}$

0,80

1,00

Ni et $\mathrm{Su}, 2001$ (CMQ,

4,02

1,22

$1,00^{\#}$

0,80

1,00 
chinois)

$\mathrm{Ni}$, Su, Chung et

Cheng, 2005 (conf. A-

3,60

0,60

$1,00^{\#}$

1,00

1,00

$P$, anglais)

$\mathrm{Ni}$, Su, Chung et

Cheng, 2005 (conf. A-

0,53

$1,00^{\#}$

1,00

1,00

$P$, anglais)

$\mathrm{Ni}$, Su, Chung et

Cheng, 2005 (conf. A-

3,78

0,78

$1,00^{\#}$

1,00

1,00

$P$, anglais)

Nor, 2007 (conf. SNA, indonésien)

4,24

1,24

1,00

1,00

1,00

Nouri, Blau et Shahid, 1995 (AOS, anglais)

4,46

1,66

0,50

1,30

1,00

Nouri et Parker, 1998 (AOS, anglais)

4,26

1,66

0,50

1,10

1,00

Otley et Pollanen,

2000 (AOS, anglais)

4,59

1,49

1,00

1,10

1,00

Parker et Kyj, 2006

(AOS, anglais)

4,06

0,86

1,00

1,20

1,00

Puspaningsih, 2003

(JAAI, indonésien)

3,36

0,71

0,75

0,90

1,00

Qi, 2010 (thèse, anglais)

3,77

0,92

0,75

1,10

1,00

Quirin, O'Bryan et

Donnelly, 2004 (AMA, anglais)

4,21

1,21

$1,00^{\#}$

1,00

1,00

Sardjito et Muthaher,

2007 (conf. SNA, indonésien)

4,16

1,26

1,00

0,90

1,00

6,41

4,41

0,00

1,00

1,00

2000 (AOS, anglais)

2,30

0,20

0,00

1,10

1,00

indonésien)

Su, 2001 (communiqué

par Ni, Su et Su, 2003)

(mémoire, chinois)

0,81

0,50

1,10

1,00

Su, 2001 (communiqué

par Ni, Su et Su, 2003)

(mémoire, chinois)

3,22

0,62

0,50

1,10

1,00

Su, 2001 (communiqué

par Ni, Su et Su, 2003)

(mémoire, chinois)

3,01

0,41

0,50

1,10

1,00

Su, 2001 (communiqué

par Ni, Su et Su, 2003)

(mémoire, chinois)

3,15

0,55

0,50

1,10

1,00

Su, 2008 (thèse, chinois)

5,02

2,02

$1,00^{\#}$

1,00

1,00

Su et Lin, 2007 (WP,

5,27

2,07

$1,00^{\#}$

1,20

1,00 


\begin{tabular}{|c|c|c|c|c|c|c|}
\hline $\begin{array}{c}\text { Subramaniam et } \\
\text { Ashkanasy, } 2001 \text { (AJM, } \\
\text { anglais) }\end{array}$ & $*$ & 4,60 & 1,40 & $1,00^{\#}$ & 1,20 & 1,00 \\
\hline $\begin{array}{c}\text { Sumarno, } 2006 \\
\text { (mémoire, indonésien) }\end{array}$ & & 3,03 & 1,23 & 0,00 & 0,80 & 1,00 \\
\hline $\begin{array}{c}\text { Syah, } 2009 \text { (mémoire, } \\
\text { indonésien) }\end{array}$ & & 2,41 & 0,41 & 0,00 & 1,00 & 1,00 \\
\hline Taylor, Abdul-Hamid et & & & & & & \\
\hline $\begin{array}{c}\text { Mohd-Sanusi, } 2008 \\
\text { (JAMAR, anglais) }\end{array}$ & & 3,75 & 1,00 & 0,75 & 1,00 & 1,00 \\
\hline $\begin{array}{l}\text { Tintri, } 2002 \text { (JEB, } \\
\text { indonésien) }\end{array}$ & & 3,55 & 0,65 & 1,00 & 0,90 & 1,00 \\
\hline $\begin{array}{c}\text { Tsui, } 2001 \text { (IJA, } \\
\text { anglais) }\end{array}$ & & 4,10 & 1,10 & 1,00 & 1,00 & 1,00 \\
\hline $\begin{array}{c}\text { Wentzel, } 2002 \text { (BRA, } \\
\text { anglais) }\end{array}$ & & 4,01 & 0,91 & 1,00 & 1,10 & 1,00 \\
\hline $\begin{array}{l}\text { Winata et Mia, } 2005 \\
\text { (IJHM, anglais) }\end{array}$ & & 3,36 & 0,91 & 0,25 & 1,20 & 1,00 \\
\hline Wu, 2004 (publié par & & & & & & \\
\hline $\begin{array}{c}\text { Chang, Chang, Chang } \\
\text { et Wu, 2007) (IJAS, } \\
\text { chinois) }\end{array}$ & $*$ & 2,98 & 0,78 & 0,00 & 1,20 & 1,00 \\
\hline Wu, 2004 (publié par & & & & & & \\
\hline $\begin{array}{c}\text { Chang, Chang, Chang } \\
\text { et Wu, 2007) (IJAS, } \\
\text { chinois) }\end{array}$ & $*$ & 2,71 & 0,51 & 0,00 & 1,20 & 1,00 \\
\hline Yahya, Ahmad et & & & & & & \\
\hline $\begin{array}{c}\text { Fatima, } 2008 \text { (IJPSM, } \\
\text { anglais) }\end{array}$ & & 4,47 & 1,37 & 1,00 & 1,10 & 1,00 \\
\hline $\begin{array}{c}\text { Yuen, } 2007 \text { (MAJ, } \\
\text { anglais) }\end{array}$ & & 5,61 & 2,66 & 0,75 & 1,20 & 1,00 \\
\hline $\begin{array}{l}\text { Zhu, Lin et Ni, } 2002 \\
\text { (PMR, anglais) }\end{array}$ & $*$ & 4,63 & 1,93 & 0,50 & 1,20 & 1,00 \\
\hline
\end{tabular}

Note : Les supports de publication sont résumés par leurs acronymes. «WP » est l'acronyme de working paper pour une étude non publiée et « conf. » rend compte du fait qu'il s'agit d'un article communiqué et non publié.

Les critères ont été fixés avant une lecture détaillée des articles afin de limiter les biais (Slavin, 1995).

\# Les articles dont le score de pertinence des échelles porte un dièse sont les articles retenus pour notre synthèse des meilleures études.

1. «Participative budgeting exists for planning and goal setting when there is environmental uncertainty; it exists for motivating subordinates when there is task uncertainty; and, it exists for coordinating interdependence when there is task interdependence. » (Shields et Shields 1998, p. 65.)

2. «Slack is defined as the amount by which a subordinate overstates his/her needs for resources to complete a task or understates his/her productive capability when given the opportunity to influence the standard against which his/her performance will be evaluated. » (Walker et Johnson 1999, p. 3.)

3. La validité externe est entendue comme la possibilité de généraliser une relation observée à une population d'individus (Calder et al. 1982, p. 240).

4. «A quantitative method of combining the results of independent studies (usually drawn from the published literature) and synthesizing summaries and conclusions which may be used to evaluate therapeutic effectiveness, plan new 
studies, etc., with application chiefly in the areas of research and medicine. » (Olkin 1995, p. 133.)

5. Pour une synthèse et une explication plus approfondies des critiques adressées aux revues de littérature classiques, le lecteur peut se référer à Slavin $(1995$, p. 9 et 10, «Limitations of narrative reviews »).

6. «Le biais de publication vient du fait que les résultats statistiquement [...] positifs et significatifs ont plus de chance d'être publiés et présentés à des congrès scientifiques que les résultats négatifs. » (Grégoire et al. 1995, p. 159, T.D.A.)

7. Les expressions « taille d'effet », « taille de l'effet » et « effet commun » sont utilisées indifféremment dans cet article pour traduire le concept anglais d'effect size.

8. Retenons que ce critère est subjectif et qu'il doit être donné, à bulletin secret, par deux individus au moins pour diminuer les biais individuels et collectifs. Cela a été réalisé pour la méta-analyse quantitative prenant en compte le score de qualité. Une discussion argumentée a suivi cette procédure de notation en double aveugle pour trouver une évaluation convenable.

9. Parmi les 80 articles entrant dans la méta-analyse quantitative, 51 études s'appuient conjointement sur ces deux échelles et seulement 11 n'en utilisent aucune des deux. Les auteurs sont conscients que le fait de s'appuyer sur un consensus est critiquable. Cependant, suivant un principe issu de la philosophie d'Aristote (Rhetoric, Book I, -350), « quand il n'y a pas de réponse scientifique disponible, l'opinion perçue (Doxa) ou admise par la majorité a valeur de vérité » (T.D.A.).

10. Pour un article, la règle de décision utilisée pour choisir le meilleur résultat est la suivante :

- si les résultats portent sur le même échantillon, nous retenons le modèle ayant le $\mathrm{R}^{2}$ (ajusté) le plus élevé ;

- si, dans le cas précédent, nous avons deux $\mathrm{R}^{2}$ (ajustés) égaux, alors nous retenons le modèle le plus simple (ayant le moins de variables explicatives selon le principe du rasoir d'Occam) ou ayant la $F$-value la plus élevée ;

- si l'échantillon global est décomposé en sous-échantillons, nous retenons la décomposition la plus fine (et le modèle ayant le $\mathrm{R}^{2}$ (ajusté) le plus élevé si besoin);

- si une régression et une analyse des chemins de causalité (ou path analysis) sont disponibles, nous retenons le résultat de la régression;

- s'il s'agit d'une path analysis, le résultat du modèle remplissant au mieux les critères suivants est retenu $: \chi^{2}$ le plus faible possible ( $p$-value $>0.05$ ), RMSEA le plus faible possible, $\mathrm{CFI} \geq 0.9, \mathrm{NFI} \geq 0.9, \mathrm{NNFI} \approx 1$ (Breaux 2004);

- $\quad$ si les statistiques de l'article sont incohérentes entre elles, le résultat est écarté ;

- lorsque les échelles de mesures des concepts sont scindées pour la mesure de sous-concepts, seuls les résultats sur l'échelle complète sont retenus ;

- $\quad$ si aucun $\mathrm{R}^{2}$ n'est disponible, le modèle possédant le plus de variables significatives est retenu.

11. L'hypothèse $\mathrm{H}_{0}$ testée est toujours celle de la nullité de l'effet de la participation budgétaire sur la performance managériale $\left(\mathrm{H}_{0}: \hat{\theta}=0\right)$.

12. Derfuss $(2009$, p. 221) a réalisé une méta-analyse quantitative sur huit études dont l'échantillon a été sélectionné aléatoirement et utilisant les échelles de Milani (1975) et Mahoney et al. $(1963,1965)$, ce qui peut être apparenté à une «synthèse des meilleures études » même si l'auteur n'a pas fait référence à cette méthode.

13. Nous remercions un des réviseurs anonymes pour nous avoir suggéré ces analyses additionnelles.

14. Nous remercions un des réviseurs anonymes pour nous avoir fait cette remarque ayant permis ces analyses additionnelles. 